A NNALES

UNIVERSITATIS MARIAE CURIE-SKŁODOWSKA

L UBLIN - P OLONIA

VOL. LXVIII, NO. 1, 2014

SECTIO A

$67-89$

\author{
MARIUSZ PLASZCZYK
}

\title{
The constructions of general connections on second jet prolongation
}

\begin{abstract}
We determine all natural operators $D$ transforming general connections $\Gamma$ on fibred manifolds $Y \rightarrow M$ and torsion free classical linear connections $\nabla$ on $M$ into general connections $D(\Gamma, \nabla)$ on the second order jet prolongation $J^{2} Y \rightarrow M$ of $Y \rightarrow M$.
\end{abstract}

1. Introduction. The concept of $r$-th order connections was firstly introduced on groupoids by C. Ehresmann in [2] and next by I. Kolář in [3] for arbitrary fibred manifolds.

Let us recall that an $r$-th order connection on a fibred manifold $p: Y \rightarrow$ $M$ is a section $\Theta: Y \rightarrow J^{r} Y$ of the $r$-jet prolongation $\beta: J^{r} Y \rightarrow Y$ of $p: Y \rightarrow M$. A general connection on $p: Y \rightarrow M$ is a first order connection $\Gamma: Y \rightarrow J^{1} Y$ or (equivalently) a lifting map

$$
\Gamma: Y \times_{M} T M \rightarrow T Y .
$$

By $\operatorname{Con}(Y \rightarrow M)$ we denote the set of all general connections on a fibred manifold $p: Y \rightarrow M$.

If $p: Y \rightarrow M$ is a vector bundle and an $r$-th order connection $\Theta: Y \rightarrow$ $J^{r} Y$ is a vector bundle morphism, then $\Theta$ is called an $r$-th order linear connection on $p: Y \rightarrow M$.

2000 Mathematics Subject Classification. 58A05, 58A20, 58A32.

Key words and phrases. General connection, classical linear connection, higher order jet prolongation, bundle functor, natural operator. 
An $r$-th order linear connection on $M$ is an $r$-th order linear connection $\Lambda: T M \rightarrow J^{r} T M$ on the tangent bundle $\pi_{M}: T M \rightarrow M$ of $M$. By $Q^{r}(M)$ we denote the set of all $r$-th order linear connections on $M$.

A classical linear connection on $M$ is a first order linear connection $\nabla: T M \rightarrow J^{1} T M$ or (equivalently) a covariant derivative $\nabla: \mathfrak{X}(M) \times$ $\mathfrak{X}(M) \rightarrow \mathfrak{X}(M)$. A classical linear connection $\nabla$ on $M$ is called torsion free if its torsion tensor $T(X, Y)=\nabla_{X} Y-\nabla_{Y} X-[X, Y]$ is equal to zero. By $Q_{\tau}(M)$ we denote the set of all torsion free classical linear connections on $M$.

Let $\mathcal{F M}$ denote the category of fibred manifolds and their fibred maps and let $\mathcal{F} \mathcal{M}_{m, n} \subset \mathcal{F} \mathcal{M}$ be the (sub)category of fibred manifolds with $m$ dimensional bases and $n$-dimensional fibres and their local fibred diffeomorphisms. Let $\mathcal{M} f_{m}$ denote the category of $m$-dimensional manifolds and their local diffeomorphisms. Let $F: \mathcal{F} \mathcal{M}_{m, n} \rightarrow \mathcal{F M}$ be a bundle functor on $\mathcal{F} \mathcal{M}_{m, n}$ of order $r$ in the sense of [4]. Let $\Gamma: Y \times_{M} T M \rightarrow T Y$ be the lifting map of a general connection on an object $p: Y \rightarrow M$ of $\mathcal{F} \mathcal{M}_{m, n}$. Let $\Lambda: T M \rightarrow J^{r} T M$ be an $r$-th order linear connection on $M$. The flow operator $\mathcal{F}$ of $F$ transforming projectable vector fields $\eta$ on $p: Y \rightarrow M$ into vector fields $\mathcal{F} \eta:=\left.\frac{\partial}{\partial t}\right|_{t=0} F\left(F l_{t}^{\eta}\right)$ on $F Y$ is of order $r$. In other words, the value $\mathcal{F} \eta(u)$ at every $u \in F_{y} Y, y \in Y$ depends only on $j_{y}^{r} \eta$. Therefore, we have the corresponding flow morphism $\tilde{\mathcal{F}}: F Y \times_{Y} J^{r} T Y \rightarrow T F Y$, which is linear with respect to $J^{r} T Y$. Moreover, $\tilde{\mathcal{F}}\left(u, j_{y}^{r} \eta\right)=\mathcal{F} \eta(u)$, where $u \in F_{y} Y, y \in Y$. Let $X^{\Gamma}$ be the $\Gamma$-lift of a vector field $X$ on $M$ to $Y$, i.e. $X^{\Gamma}$ is a projectable vector field on $p: Y \rightarrow M$ defined by $X^{\Gamma}(y)=\Gamma(y, X(x)), y \in$ $Y_{x}, x=p(y) \in M$. Then the connection $\Gamma$ can be extended to a morphism $\tilde{\Gamma}: Y \times_{M} J^{r} T M \rightarrow J^{r} T Y$ by the following formula $\tilde{\Gamma}\left(y, j_{x}^{r} X\right)=j_{y}^{r}\left(X^{\Gamma}\right)$. By applying $\mathcal{F}$, we obtain a map $\mathcal{F}(\tilde{\Gamma}): F Y \times_{M} J^{r} T M \rightarrow T F Y$ defined by $\mathcal{F}(\tilde{\Gamma})\left(u, j_{x}^{r} X\right)=\tilde{\mathcal{F}}\left(u, j_{y}^{r}\left(X^{\Gamma}\right)\right)=\mathcal{F} X^{\Gamma}(u)$. Further the composition

$$
\mathcal{F}(\Gamma, \Lambda):=\mathcal{F}(\tilde{\Gamma}) \circ\left(i d_{F Y} \times \Lambda\right): F Y \times_{M} T M \rightarrow T F Y
$$

is the lifting map of a general connection on $F Y \rightarrow M$. The connection $\mathcal{F}(\Gamma, \Lambda)$ is called $F$-prolongation of $\Gamma$ with respect to $\Lambda$ and was discovered by I. Kolář [5].

Let $\nabla$ be a torsion free classical linear connection on $M$. For every $x \in M$, the connection $\nabla$ determines the exponential map $\exp _{x}^{\nabla}: T_{x} M \rightarrow M$ (of $\nabla$ in $x$ ), which is diffeomorphism of some neighbourhood of the zero vector at $x$ onto some neighbourhood of $x$. Every vector $v \in T_{x} M$ can be extended to a vector field $\tilde{v}$ on a vector space $T_{x} M$ by $\tilde{v}(w)=\frac{\partial}{\partial t} \mid t=0[w+t v]$. Then we can construct an $r$-th order linear connection $E_{r}(\nabla): T M \rightarrow J^{r} T M$, which is given by $E_{r}(\nabla)(v)=j_{x}^{r}\left(\left(\exp _{x}^{\nabla}\right)_{*} \tilde{v}\right)$. This connection is called an exponential extension of $\nabla$ and was presented by W. Mikulski in [9]. Another equivalent definition (for corresponding principal connections in the $r$-frame bundles) 
of the exponential extension was independently introduced by I. Kolář in [6]. Hence given a general connection $\Gamma$ on $Y \rightarrow M$ and a torsion free classical linear connection $\nabla$ on $M$, we have the general connection

$$
\mathcal{F}(\Gamma, \nabla):=\mathcal{F}\left(\Gamma, E_{r}(\nabla)\right): F Y \times_{M} T M \rightarrow T F Y .
$$

The canonical character of construction of this connection can be described by means of the concept of natural operators. The general concept of natural operators can be found in [4]. In particular, we have the following definitions.

Definition 1. Let $F: \mathcal{F} \mathcal{M}_{m, n} \rightarrow \mathcal{F} \mathcal{M}$ be a bundle functor of order $r$ on a category $\mathcal{F M}_{m, n}$. An $\mathcal{F} \mathcal{M}_{m, n}$-natural operator $D: J^{1} \times Q_{\tau}(\mathcal{B}) \rightsquigarrow J^{1}(F \rightarrow$ $\mathcal{B})$ transforming general connections $\Gamma$ on fibred manifolds $p: Y \rightarrow M$ and torsion free classical linear connections $\nabla$ on $M$ into general connections $D(\Gamma, \nabla): F Y \rightarrow J^{1} F Y$ on $F Y \rightarrow M$ is a system of regular operators $D_{Y}: \operatorname{Con}(Y \rightarrow M) \times Q_{\tau}(M) \rightarrow \operatorname{Con}(F Y \rightarrow M),(p: Y \rightarrow$ $M) \in \operatorname{Obj}\left(\mathcal{F} \mathcal{M}_{m, n}\right)$ satisfying the $\mathcal{F} \mathcal{M}_{m, n}$-invariance condition: for any $\Gamma \in \operatorname{Con}(Y \rightarrow M), \Gamma_{1} \in \operatorname{Con}\left(Y_{1} \rightarrow M_{1}\right), \nabla \in Q_{\tau}(M)$ and $\nabla_{1} \in Q_{\tau}\left(M_{1}\right)$ such that if $\Gamma$ is $f$-related to $\Gamma_{1}$ by an $\mathcal{F M}_{m, n}$-map $f: Y \rightarrow Y_{1}$ covering $\underline{f}: M \rightarrow M_{1}$ (i.e. $J^{1} f \circ \Gamma=\Gamma_{1} \circ f$ ) and $\nabla$ is $\underline{f}$-related to $\nabla_{1}$ (i.e. $\left.J^{1} T \underline{f} \circ \nabla=\nabla_{1} \circ T \underline{f}\right)$, then $D_{Y}(\Gamma, \nabla)$ is $F f$-related to $D_{Y_{1}}\left(\Gamma_{1}, \nabla_{1}\right)$ (i.e. $\left.J^{1} F f \circ D_{Y}(\Gamma, \nabla)=\bar{D}_{Y_{1}}\left(\Gamma_{1}, \nabla_{1}\right) \circ F f\right)$. Equivalently the $\mathcal{F} \mathcal{M}_{m, n}$-invariance means that for any $\Gamma \in \operatorname{Con}(Y \rightarrow M), \Gamma_{1} \in \operatorname{Con}\left(Y_{1} \rightarrow M_{1}\right), \nabla \in Q_{\tau}(M)$ and $\nabla_{1} \in Q_{\tau}\left(M_{1}\right)$ if diagrams
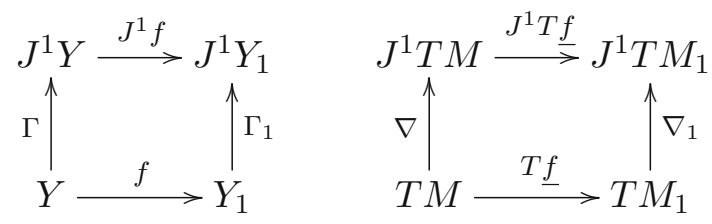

commute for a $\mathcal{F} \mathcal{M}_{m, n}$-map $f: Y \rightarrow Y_{1}$ covering $f: M \rightarrow M_{1}$, then the diagram

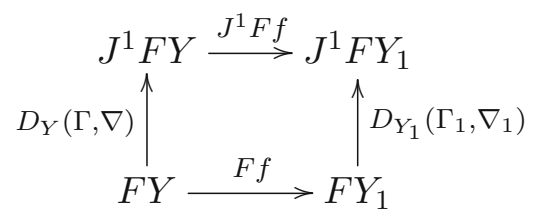

commutes. We say that the operator $D_{Y}$ is regular if it transforms smoothly parametrized families of connections into smoothly parametrized ones.

Definition 2. A $\mathcal{M} f_{m}$-natural operator $A: Q_{\tau} \rightsquigarrow Q^{r}$ extending torsion free classical linear connections $\nabla$ on $m$-dimensional manifolds $M$ into $r$-th order linear connections $A(\nabla): T M \rightarrow J^{r} T M$ on $M$ is a system of regular 
operators $A_{M}: Q_{\tau}(M) \rightarrow Q^{r}(M), M \in \operatorname{Obj}\left(\mathcal{M} f_{m}\right)$ satisfying the $\mathcal{M} f_{m^{-}}$ invariance condition: if $\nabla \in Q_{\tau}(M)$ and $\nabla_{1} \in Q_{\tau}\left(M_{1}\right)$ are $f$-related by a $\mathcal{M} f_{m}$-map $f: M \rightarrow M_{1}$ (i.e. $J^{1} T f \circ \nabla=\nabla_{1} \circ T f$ ), then $A(\nabla)$ and $A\left(\nabla_{1}\right)$ are $f$-related, too (i.e. $J^{r} T f \circ A(\nabla)=A\left(\nabla_{1}\right) \circ T f$ ). In other words, the $\mathcal{M} f_{m}$-invariance means that if for any $\nabla \in Q_{\tau}(M), \nabla_{1} \in Q_{\tau}\left(M_{1}\right)$ the diagram

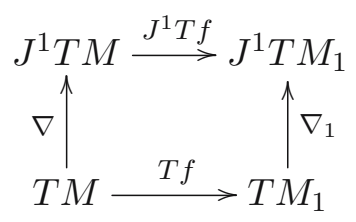

commutes for a $\mathcal{M} f_{m}$-map $f: M \rightarrow M_{1}$, then the following diagram

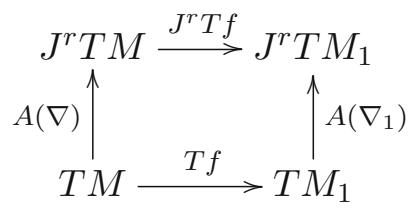

commutes, too. The regularity means that every $A_{M}$ transforms smoothly parametrized families of connections into smoothly parametrized ones.

Thus the construction $\mathcal{F}(\Gamma, \Lambda)$ can be considered as the $\mathcal{F} \mathcal{M}_{m, n}$-natural operator $\mathcal{F}: J^{1} \times Q_{\tau}(\mathcal{B}) \rightsquigarrow J^{1}(F \rightarrow \mathcal{B})$. Similarly, the correspondence $E_{r}: Q_{\tau} \rightsquigarrow Q^{r}$ is the $\mathcal{M} f_{m}$-natural operator.

In [4], the authors described all $\mathcal{F} \mathcal{M}_{m, n}$-natural operators $D: J^{1} \times Q_{\tau}(\mathcal{B})$ $\rightsquigarrow J^{1}(F \rightarrow \mathcal{B})$ for a bundle functor $F=J^{1}: \mathcal{F} \mathcal{M}_{m, n} \rightarrow \mathcal{F} \mathcal{M}$. They constructed an additional $\mathcal{F} \mathcal{M}_{m, n}$-natural operator $P$ and proved that all $\mathcal{F} \mathcal{M}_{m, n}$-natural operators $D: J^{1} \times Q_{\tau}(\mathcal{B}) \rightsquigarrow J^{1}\left(J^{1} \rightarrow \mathcal{B}\right)$ form the one parameter family $t P+(1-t) \mathcal{J}^{1}, t \in \mathbf{R}$.

In this paper we determine all $\mathcal{F} \mathcal{M}_{m, n}$-natural operators $D: J^{1} \times Q_{\tau}(\mathcal{B})$ $\rightsquigarrow J^{1}\left(J^{2} \rightarrow \mathcal{B}\right)$. We assume that all manifolds and maps are smooth, i.e. of class $C^{\infty}$.

2. Quasi-normal fibred coordinate systems. Let $\Gamma: Y \rightarrow J^{1} Y$ be a general connection on a fibred manifold $p: Y \rightarrow M$ with $\operatorname{dim}(M)=m$ and $\operatorname{dim}(Y)=m+n, \nabla$ be a torsion free classical linear connection on $M$ and $y_{0} \in Y$ be a point with $x_{0}=p\left(y_{0}\right) \in M$.

In [8] W. Mikulski presented a concept of $\left(\Gamma, \nabla, y_{0}, r\right)$-quasi-normal fibred coordinate systems on $Y$ for any $r$. For $r=3$ this concept can be equivalently defined in the following way.

Definition 3. A $\left(\Gamma, \nabla, y_{0}, 3\right)$-quasi-normal fibred coordinate system on $Y$ is a fibred chart $\psi$ on $Y$ with $\psi\left(y_{0}\right)=(0,0) \in \mathbf{R}^{m, n}$ covering a chart $\psi$ on $M$ with centre $x_{0}$ if the map $i d_{\mathbf{R}^{m}}$ is a $\underline{\psi}_{*} \nabla$-normal coordinate system with 
centre $0 \in \mathbf{R}^{m}$ and an element $j_{(0,0)}^{2}\left(\psi_{*} \Gamma\right) \in J_{(0,0)}^{2}\left(J^{1} \mathbf{R}^{m, n} \rightarrow \mathbf{R}^{m, n}\right)$ is of the form

$$
\begin{aligned}
j_{(0,0)}^{2}\left(\psi_{*} \Gamma\right) & =j_{(0,0)}^{2}\left(\Gamma_{0}+\sum_{i, j, k=1}^{m} \sum_{p=1}^{n} a_{k i j}^{p} x^{k} x^{i} d x^{j} \otimes \frac{\partial}{\partial y^{p}}\right. \\
& \left.+\sum_{i, j=1}^{m} \sum_{p, q=1}^{n} b_{q i j}^{p} y^{q} x^{i} d x^{j} \otimes \frac{\partial}{\partial y^{p}}+\sum_{i, j=1}^{m} \sum_{p=1}^{n} c_{i j}^{p} x^{i} d x^{j} \otimes \frac{\partial}{\partial y^{p}}\right)
\end{aligned}
$$

for some (uniquely determined) real numbers $a_{k i j}^{p}, b_{q i j}^{p}$ and $c_{i j}^{p}$ satisfying

$$
\begin{aligned}
& a_{k i j}^{p}-a_{i k j}^{p}=0 \\
& a_{k i j}^{p}+a_{k j i}^{p}+a_{i k j}^{p}+a_{i j k}^{p}+a_{j i k}^{p}+a_{j k i}^{p}=0 \\
& b_{q i j}^{p}+b_{q j i}^{p}=0 \\
& c_{i j}^{p}+c_{j i}^{p}=0,
\end{aligned}
$$

where $\Gamma_{0}=\sum_{i=1}^{m} d x^{i} \otimes \frac{\partial}{\partial x^{i}}$ is the trivial general connection on $\mathbf{R}^{m, n}$ and $x^{1}, \ldots, x^{m}, y^{1}, \ldots, y^{n}$ are the usual fibred coordinates on $\mathbf{R}^{m, n}$.

In [8] W. Mikulski proved an important proposition ([8], Proposition 2.2) concerning $\left(\Gamma, \nabla, y_{0}, r\right)$-quasi-normal fibred coordinate systems. Below we recall this result for $r=3$. A fibred-fibred manifold version of Proposition 2.2 from [8] for $r=1$ is presented in [7].

Proposition 1. Let $\Gamma: Y \rightarrow J^{1} Y$ be a general connection on a fibred manifold $p: Y \rightarrow M$ with $\operatorname{dim}(M)=m$ and $\operatorname{dim}(Y)=m+n, \nabla$ be a torsion free classical linear connection on $M$ and $y_{0} \in Y$ be a point with $x_{0}=p\left(y_{0}\right) \in M$. Then:

(i) There exists a $\left(\Gamma, \nabla, y_{0}, 3\right)$-quasi-normal fibred coordinate system $\psi$ on $Y$. (ii) If $\psi^{1}$ is another $\left(\Gamma, \nabla, y_{0}, 3\right)$-quasi-normal fibred coordinate system, then

$$
j_{y_{0}}^{3} \psi^{1}=j_{y_{0}}^{3}((B \times H) \circ \psi)
$$

for a linear map $B \in G L(m)$ and diffeomorphism $H: \boldsymbol{R}^{n} \rightarrow \boldsymbol{R}^{n}$ preserving 0 .

From the proof of Proposition 2.2 from [8] it follows that $(B \times H) \circ \psi$ is a $\left(\Gamma, \nabla, y_{0}, 3\right)$-quasi-normal fibred coordinate system for any $B \in G L(m)$ and any diffeomorphism $H: \mathbf{R}^{n} \rightarrow \mathbf{R}^{n}$ preserving 0 . In other words, the $\mathcal{F} \mathcal{M}_{m, n}$-maps of the form $B \times H$ for $B \in G L(m)$ and diffeomorphisms $H: \mathbf{R}^{n} \rightarrow \mathbf{R}^{n}$ preserving $0 \in \mathbf{R}^{n}$ transform quasi-normal fibred coordinate systems into quasi-normal ones.

From now on we will usually work in $\left(\Gamma, \nabla, y_{0}, 3\right)$-quasi-normal fibred coordinates for considered $\Gamma$ and $\nabla$. If coordinates are not necessarily quasinormal, the reader will be informed. 
3. Constructions of connections. Let $\Gamma: Y \rightarrow J^{1} Y$ be a general connection on an $\mathcal{F M}_{m, n}$-object $p: Y \rightarrow M$ and let $\nabla: T M \rightarrow J^{1} T M$ be a torsion free classical linear connection on $M$.

Example 1. Let $A: Q_{\tau} \rightsquigarrow Q^{2}$ be a $\mathcal{M} f_{m}$-natural operator and let $\Lambda=$ $A(\nabla): T M \rightarrow J^{2} T M$ be a second order linear connection on $M$ canonically depending on $\nabla$. Then from Introduction for a functor $F=J^{2}$, we have a general connection

$$
\mathcal{J}_{(A)}^{2}(\Gamma, \nabla):=\mathcal{J}^{2}(\Gamma, A(\nabla)): J^{2} Y \rightarrow J^{1} J^{2} Y
$$

on $J^{2} Y \rightarrow M$ canonically depending on $\Gamma$ and $\nabla$.

Because of the canonical character of the construction $\mathcal{J}_{(A)}^{2}(\Gamma, \nabla)$ we obtain the following proposition.

Proposition 2. The family $\mathcal{J}_{(A)}^{2}: J^{1} \times Q_{\tau}(\mathcal{B}) \rightsquigarrow J^{1}\left(J^{2} \rightarrow \mathcal{B}\right)$ of functions

$$
\mathcal{J}_{(A)}^{2}: \operatorname{Con}(Y \rightarrow M) \times Q_{\tau}(M) \rightarrow \operatorname{Con}\left(J^{2} Y \rightarrow M\right)
$$

for all $\mathcal{F} \mathcal{M}_{m, n}$-objects $Y \rightarrow M$ is an $\mathcal{F} \mathcal{M}_{m, n}$-natural operator.

Example 2. For every torsion free classical linear connection $\nabla$ on a manifold $M$ we have a canonical vector bundle isomorphism $\psi_{\nabla}: J^{2} T M \rightarrow$ $\oplus_{k=0}^{2} S^{k} T^{*} M \otimes T M$ given by a formula

$$
\psi_{\nabla}(\tau)=\oplus_{k=0}^{2} S^{k} T_{0}^{*} \varphi^{-1} \otimes T_{0} \varphi^{-1}\left(I\left(J^{2} T \varphi(\tau)\right)\right),
$$

where $\tau \in J_{x}^{2} T M, x \in M, \varphi$ is a $\nabla$-normal coordinate system on $M$ with centre $x$ and $I: J_{0}^{2} T \mathbf{R}^{m} \rightarrow \oplus_{k=0}^{2} S^{k} T_{0}^{*} \mathbf{R}^{m} \otimes T_{0} \mathbf{R}^{m}$ is the usual identification.

In the main result of [9], W. Mikulski showed that $\mathcal{M} f_{m}$-natural operators $A: Q_{\tau} \rightsquigarrow Q^{2}$ are in bijection with $\mathcal{M} f_{m}$-natural operators $A_{0} \equiv 0: Q_{\tau} \rightsquigarrow$ $T^{*} \otimes T, A_{1}: Q_{\tau} \rightsquigarrow T^{*} \otimes T^{*} \otimes T$ and $A_{2}: Q_{\tau} \rightsquigarrow T^{*} \otimes S^{2} T^{*} \otimes T$. In other words, the second order linear connections $\Lambda=A(\nabla): T M \rightarrow J^{2} T M$ on $M$ canonically depending on $\nabla$ are in bijection with the tensor fields $A_{0}(\nabla) \equiv$ $0: M \rightarrow T^{*} M \otimes T M, A_{1}(\nabla): M \rightarrow T^{*} M \otimes T^{*} M \otimes T M$ and $A_{2}(\nabla): M \rightarrow$ $T^{*} M \otimes S^{2} T^{*} M \otimes T M$ on $M$ canonically depending on $\nabla$.

Now by means of $\psi_{\nabla}, A_{1}(\nabla) \equiv 0$ and $A_{2}(\nabla)$ we can define a second order linear connection $A(\nabla): T M \rightarrow J^{2} T M$ on $M$ by

$$
A(\nabla)(v)=\psi_{\nabla}^{-1}\left(v, 0,<A_{2}(\nabla)(x), v>\right), v \in T_{x} M, x \in M
$$

In particular, for $A_{2}(\nabla) \equiv 0: M \rightarrow T^{*} M \otimes S^{2} T^{*} M \otimes T M$ we obtain

$$
A_{2}^{\exp }(\nabla)(v)=\psi_{\nabla}^{-1}(v, 0,0): T M \rightarrow J^{2} T M,
$$

On the other hand, from [9] it follows that

$$
A_{2}^{\exp }(\nabla)(v)=E_{2}(\nabla)(v) .
$$

It means that $A_{2}^{\exp }(\nabla)$ is the second order exponential extension of $\nabla$. 
Finally, in the accordance with Example 1 we have a general connection

$$
\mathcal{J}_{\left(A_{2}^{\text {exp }}\right)}^{2}(\Gamma, \nabla):=\mathcal{J}^{2}\left(\Gamma, A_{2}^{e x p}(\nabla)\right): J^{2} Y \rightarrow J^{1} J^{2} Y
$$

on $J^{2} Y \rightarrow M$ canonically depending on $\Gamma$ and $\nabla$.

Example 3. Let $\rho \in\left(J^{2} Y\right)_{y_{0}}, y_{0} \in Y_{x_{0}}, x_{0} \in M$ and consider a $\left(\Gamma, \nabla, y_{0}, 3\right)$ quasi-normal fibred coordinate system $\psi$ on $Y$. Then

$$
\begin{aligned}
j_{(0,0)}^{2}\left(\psi_{*} \Gamma\right)= & j_{(0,0)}^{2}\left(\Gamma_{0}+\sum_{i, j, k=1}^{m} \sum_{p=1}^{n} a_{k i j}^{p} x^{k} x^{i} d x^{j} \otimes \frac{\partial}{\partial y^{p}}\right. \\
& \left.+\sum_{i, j=1}^{m} \sum_{p, q=1}^{n} b_{q i j}^{p} y^{q} x^{i} d x^{j} \otimes \frac{\partial}{\partial y^{p}}+\sum_{i, j=1}^{m} \sum_{p=1}^{n} c_{i j}^{p} x^{i} d x^{j} \otimes \frac{\partial}{\partial y^{p}}\right)
\end{aligned}
$$

for unique real numbers $a_{k i j}^{p}, b_{q i j}^{p}$ and $c_{i j}^{p}$ satisfying (2). Denote

$$
\begin{aligned}
\Gamma^{[1]} & =\Gamma_{0}+\sum_{i, j, k=1}^{m} \sum_{p=1}^{n} a_{k i j}^{p} x^{k} x^{i} d x^{j} \otimes \frac{\partial}{\partial y^{p}}, \\
\Gamma^{[2]} & =\Gamma_{0}+\sum_{i, j=1}^{m} \sum_{p, q=1}^{n} b_{q i j}^{p} y^{q} x^{i} d x^{j} \otimes \frac{\partial}{\partial y^{p}}+\sum_{i, j=1}^{m} \sum_{p=1}^{n} c_{i j}^{p} x^{i} d x^{j} \otimes \frac{\partial}{\partial y^{p}} .
\end{aligned}
$$

Now we define general connections $\mathcal{J}_{[1]}^{2}(\Gamma, \nabla): J^{2} Y \rightarrow J^{1} J^{2} Y$ and $\mathcal{J}_{[2]}^{2}(\Gamma, \nabla)$ : $J^{2} Y \rightarrow J^{1} J^{2} Y$ on $J^{2} Y \rightarrow M$ by

$$
\begin{aligned}
& \mathcal{J}_{[1]}^{2}(\Gamma, \nabla)(\rho):=J^{1} J^{2}\left(\psi^{-1}\right)\left(\mathcal{J}_{\left(A^{\exp }\right)}^{2}\left(\Gamma^{[1]}, \nabla^{0}\right)\left(J^{2} \psi(\rho)\right)\right), \\
& \mathcal{J}_{[2]}^{2}(\Gamma, \nabla)(\rho):=J^{1} J^{2}\left(\psi^{-1}\right)\left(\mathcal{J}_{\left(A^{\exp }\right)}^{2}\left(\Gamma^{[2]}, \nabla^{0}\right)\left(J^{2} \psi(\rho)\right)\right),
\end{aligned}
$$

where $\nabla^{0}$ is the usual flat classical linear connection on $\mathbf{R}^{m}$.

Because of the canonical character of the construction $\mathcal{J}_{[i]}^{2}(\Gamma, \nabla)$ for $i=$ 1,2 we have the following proposition.

Proposition 3. The family $\mathcal{J}_{[i]}^{2}: J^{1} \times Q_{\tau}(\mathcal{B}) \rightsquigarrow J^{1}\left(J^{2} \rightarrow \mathcal{B}\right)$ of functions

$$
\mathcal{J}_{[i]}^{2}: \operatorname{Con}(Y \rightarrow M) \times Q_{\tau}(M) \rightarrow \operatorname{Con}\left(J^{2} Y \rightarrow M\right)
$$

for all $\mathcal{F} \mathcal{M}_{m, n}$-objects $Y \rightarrow M$ is an $\mathcal{F} \mathcal{M}_{m, n}$-natural operator.

4. The main result. We can consider the first jet prolongation functor $J^{1}$ as an affine bundle functor on the category $\mathcal{F} \mathcal{M}_{m, n}$. The corresponding vector bundle functor is $T^{*} \mathcal{B} \otimes V$, where $\mathcal{B}: \mathcal{F} \mathcal{M}_{m, n} \rightarrow \mathcal{M} f_{m}$ is a base functor and $V$ is a vertical tangent functor. For this reason, for any fibred manifold $p: Y \rightarrow M$ from the category $\mathcal{F} \mathcal{M}_{m, n}$, the first jet prolongation $J^{1} Y \rightarrow Y$ is the affine bundle with the corresponding vector bundle $T^{*} M \otimes$ $V Y$. Therefore, $J^{1} J^{2} Y \rightarrow J^{2} Y$ is the affine bundle with corresponding vector bundle $T^{*} M \otimes V J^{2} Y$. Thus the set of all $\mathcal{F} \mathcal{M}_{m, n}$-natural operators $D: J^{1} \times Q_{\tau}(\mathcal{B}) \rightsquigarrow J^{1}\left(J^{2} \rightarrow \mathcal{B}\right)$ possesses the affine space structure. 
The following theorem classifies all $\mathcal{F} \mathcal{M}_{m, n}$-natural operators $D: J^{1} \times$ $Q_{\tau}(\mathcal{B}) \rightsquigarrow J^{1}\left(J^{2} \rightarrow \mathcal{B}\right)$.

Theorem 1. Let $D: J^{1} \times Q_{\tau}(\mathcal{B}) \rightsquigarrow J^{1}\left(J^{2} \rightarrow \mathcal{B}\right)$ be an $\mathcal{F M}_{m, n}$-natural operator transforming general connections $\Gamma: Y \rightarrow J^{1} Y$ on $\mathcal{F} \mathcal{M}_{m, n}$-objects $Y \rightarrow M$ and torsion free classical linear connections $\nabla$ on $M$ into general connections $D(\Gamma, \nabla): J^{2} Y \rightarrow J^{1} J^{2} Y$ on $J^{2} Y \rightarrow M$.

If $m \geq 2$, then there exist uniquely determined real numbers $t_{0}, t_{1}, t_{2}$ with $t_{0}+t_{1}+t_{2}=1$ and $\mathcal{M} f_{m}$-natural operator $A: Q_{\tau} \rightsquigarrow Q^{2}$ transforming torsion free classical linear connections $\nabla$ on $\mathcal{M} f_{m}$-objects $M$ into second order linear connections $A(\nabla): T M \rightarrow J^{2} T M$ on $M$ such that

$$
D(\Gamma, \nabla)=t_{0} \mathcal{J}_{(A)}^{2}(\Gamma, \nabla)+t_{1} \mathcal{J}_{[1]}^{2}(\Gamma, \nabla)+t_{2} \mathcal{J}_{[2]}^{2}(\Gamma, \nabla)
$$

for any $\mathcal{F M}_{m, n}$-object $Y \rightarrow M$, any general connection $\Gamma$ on $Y \rightarrow M$ and any torsion free classical linear connection $\nabla$ on $M$. Besides, if $t_{0} \neq 0$, then $A$ is uniquely determined (else $A$ can be arbitrary).

In the case $m=1, D=\mathcal{J}^{2}$.

In the proof we use methods for finding natural operators presented in [4] and lemmas from [1].

Proof. Let $x^{i}, y^{p}$ be the usual fibred coordinates on $\mathbf{R}^{m, n}$,

$$
y_{i}^{p}=\frac{\partial y^{p}}{\partial x^{i}}, \quad y_{i j}^{p}=y_{j i}^{p}=\frac{\partial^{2} y^{p}}{\partial x^{i} \partial x^{j}}
$$

be the additional coordinates on $J^{2} \mathbf{R}^{m, n}$ and

$$
Y^{p}=d y^{p}, \quad Y_{i}^{p}=d y_{i}^{p}, \quad Y_{i j}^{p}=Y_{j i}^{p}=d y_{i j}^{p}
$$

be the essential coordinates on the vertical bundle $V J^{2} \mathbf{R}^{m, n}$ of $J^{2} \mathbf{R}^{m, n} \rightarrow$ $\mathbf{R}^{m}$, where $i, j=1, \ldots, m$ and $p=1, \ldots, n$.

On $J_{0}^{2}\left(J^{1} \mathbf{R}^{m, n}\right)$ we have the coordinates

$$
\begin{aligned}
\Gamma_{i}^{p}, \quad \Gamma_{i j}^{p} & =\frac{\partial \Gamma_{i}^{p}}{\partial x^{j}}, \quad \Gamma_{i q}^{p}=\frac{\partial \Gamma_{i}^{p}}{\partial y^{q}}, \quad \Gamma_{i j k}^{p}=\frac{\partial^{2} \Gamma_{i}^{p}}{\partial x^{j} \partial x^{k}}, \\
\Gamma_{i q r}^{p} & =\frac{\partial^{2} \Gamma_{i}^{p}}{\partial y^{q} \partial y^{r}}, \quad \Gamma_{i j q}^{p}=\frac{\partial^{2} \Gamma_{i}^{p}}{\partial x^{j} \partial y^{q}} .
\end{aligned}
$$

The standard coordinates on $J_{0}^{1}\left(Q_{\tau}\left(\mathbf{R}^{m}\right)\right)$ are $\nabla_{j k}^{i}=\nabla_{k j}^{i}$ and $\nabla_{j k l}^{i}=\nabla_{k j l}^{i}$, where $i, j, k, l=1, \ldots, m$.

Let $\omega_{k}$ be the usual coordinates on $T^{*} \mathbf{R}^{m}$. Then the induced coordinates on the tensor product $\left(T^{*} \mathbf{R}^{m} \otimes V J^{2} \mathbf{R}^{m, n}\right)_{0}$ are

$$
Z_{k}^{p}=Y^{p} \omega_{k}, \quad Z_{i ; k}^{p}=Y_{i}^{p} \omega_{k}, \quad Z_{i j ; k}^{p}=Y_{i j}^{p} \omega_{k} .
$$

Let $D: J^{1} \times Q_{\tau}(\mathcal{B}) \rightsquigarrow J^{1}\left(J^{2} \rightarrow \mathcal{B}\right)$ be an $\mathcal{F} \mathcal{M}_{m, n}$-natural operator transforming general connections $\Gamma: Y \rightarrow J^{1} Y$ on $\mathcal{F} \mathcal{M}_{m, n^{-}}$objects $Y \rightarrow M$ and 
torsion free classical linear connections $\nabla$ on $M$ into general connections $D(\Gamma, \nabla): J^{2} Y \rightarrow J^{1} J^{2} Y$ on $J^{2} Y \rightarrow M$.

Since $J^{1} J^{2} Y \rightarrow J^{2} Y$ is the affine bundle with the corresponding vector bundle $T^{*} M \otimes V J^{2} Y$, we have the corresponding $\mathcal{F} \mathcal{M}_{m, n}$-natural operator

$$
\Delta_{D}: J^{1} \times Q_{\tau}(\mathcal{B}) \rightsquigarrow\left(J^{2}, T^{*} \mathcal{B} \otimes V J^{2}\right) .
$$

It transforms a general connection $\Gamma: Y \rightarrow J^{1} Y$ on an $\mathcal{F} \mathcal{M}_{m, n}$-object $Y \rightarrow$ $M$ and a torsion free classical linear connection $\nabla$ on $M$ into a fibred map

$$
\Delta_{D}(\Gamma, \nabla):=D(\Gamma, \nabla)-\mathcal{J}_{\left(A_{2}^{e x p}\right)}^{2}(\Gamma, \nabla): J^{2} Y \rightarrow T^{*} M \otimes V J^{2} Y .
$$

Of course, the operator $D$ is fully determined by $\Delta_{D}$ as $D(\Gamma, \nabla)=\Delta_{D}(\Gamma, \nabla)$ $+\mathcal{J}_{\left(A_{2}^{\text {exp }}\right)}^{2}(\Gamma, \nabla)$ for every $\Gamma \in \operatorname{Con}(Y \rightarrow M), \nabla \in Q_{\tau}(M)$. In other words $D=\Delta_{D}+\mathcal{J}_{\left(A_{2}^{e x p}\right)}^{2}$, so it is sufficient to investigate the operator $\Delta_{D}$.

Using the invariance of $\Delta_{D}$ with respect to the homotheties $\psi_{t}=t i d_{\mathbf{R}^{m, n}}$ covering $\underline{\psi}_{t}=t i d_{\mathbf{R}^{m}}$ for $t>0$, we have the homogeneous conditions

$$
\begin{aligned}
\left(T^{*}\left(t i d_{\mathbf{R}^{m}}\right)\right. & \left.\otimes V J^{2}\left(t i d_{\mathbf{R}^{m, n}}\right)\right)\left(\Delta_{D}(\Gamma, \nabla)(\rho)\right) \\
& =\left(\Delta_{D}\left(\left(t i d_{\mathbf{R}^{m, n}}\right)_{*} \Gamma,\left(t i d_{\mathbf{R}^{m}}\right)_{*} \nabla\right)\right)\left(J^{2}\left(t i d_{\mathbf{R}^{m, n}}\right)(\rho)\right)
\end{aligned}
$$

for any general connection $\Gamma$ on $\mathbf{R}^{m, n}$, any torsion free classical linear connection $\nabla$ on $\mathbf{R}^{m}$ and any $\rho \in\left(J^{2} \mathbf{R}^{m, n}\right)_{(0,0)}$. Using the general theory and the above local coordinates, the above condition can be written as the system of homogeneous conditions. Now, by the non-linear Peetre theorem [4] we obtain that the operator $\Delta_{D}$ is of finite order $r$ in $\Gamma$ and of order $s$ in $\nabla$. Having the natural operator $\Delta_{D}$ of order $r$ in $\Gamma$ and of finite order $s$ in $\nabla$, we shall deduce that $r=2$ and $s=1$.

The operators $\Delta_{D}$ of order 2 in $\Gamma$ and of order 1 in $\nabla$ are in bijection with $G_{m, n}^{3}$-invariant maps of standard fibres $f: S_{1} \times \Lambda \times S_{0} \rightarrow Z$ over $\underline{f}=i d_{S_{0}}$, where $S_{1}=J_{0}^{2}\left(J^{1} \mathbf{R}^{m, n}\right), \Lambda=J_{0}^{1}\left(Q_{\tau}\left(\mathbf{R}^{m}\right)\right), S_{0}=J_{0}^{2} \mathbf{R}^{m, n}, Z=$ $\left(T^{*} \mathbf{R}^{m} \otimes V J^{2} \mathbf{R}^{m, n}\right)_{0}$. This map is of the form

$$
\begin{aligned}
Z_{k}^{p} & =f_{k}^{p}\left(\Gamma_{i}^{p}, \Gamma_{i j}^{p}, \Gamma_{i q}^{p}, \Gamma_{i j k}^{p}, \Gamma_{i q r}^{p}, \Gamma_{i j q}^{p}, \nabla_{j k}^{i}, \nabla_{j k l}^{i}, y_{i}^{p}, y_{i j}^{p}\right) \\
Z_{i ; k}^{p} & =f_{i ; k}^{p}\left(\Gamma_{i}^{p}, \Gamma_{i j}^{p}, \Gamma_{i q}^{p}, \Gamma_{i j k}^{p}, \Gamma_{i q r}^{p}, \Gamma_{i j q}^{p}, \nabla_{j k}^{i}, \nabla_{j k l}^{i}, y_{i}^{p}, y_{i j}^{p}\right) \\
Z_{i j ; k}^{p} & =f_{i j ; k}^{p}\left(\Gamma_{i}^{p}, \Gamma_{i j}^{p}, \Gamma_{i q}^{p}, \Gamma_{i j k}^{p}, \Gamma_{i q r}^{p}, \Gamma_{i j q}^{p}, \nabla_{j k}^{i}, \nabla_{j k l}^{i}, y_{i}^{p}, y_{i j}^{p}\right) .
\end{aligned}
$$

The group $G_{m, n}^{3}$ acts on the standard fibre $S_{0}$ in the form

$$
\begin{aligned}
\bar{y}_{i}^{p}= & a_{q}^{p} y_{j}^{q} \tilde{a}_{i}^{j}+a_{j}^{p} \tilde{a}_{i}^{j} \\
\bar{y}_{i j}^{p}= & a_{q}^{p} y_{k l}^{q} \tilde{a}_{i}^{k} \tilde{a}_{j}^{l}+a_{q r}^{p} y_{k}^{q} y_{l}^{r} \tilde{a}_{i}^{k} \tilde{a}_{j}^{l}+a_{q k}^{p} y_{l}^{q} \tilde{a}_{i}^{k} \tilde{a}_{j}^{l}+a_{q l}^{p} y_{k}^{q} \tilde{a}_{i}^{k} \tilde{a}_{j}^{l} \\
& +a_{q}^{p} y_{k}^{q} \tilde{a}_{i j}^{k}+a_{k}^{p} \tilde{a}_{i j}^{k}+a_{k l}^{p} \tilde{a}_{i}^{k} \tilde{a}_{j}^{l}
\end{aligned}
$$


and on the fibre $S_{1}$ by the formula

$$
\begin{aligned}
\bar{\Gamma}_{i}^{p}= & a_{q}^{p} \Gamma_{j}^{q} \tilde{a}_{i}^{j}+a_{j}^{p} \tilde{a}_{i}^{j} \\
\bar{\Gamma}_{i j}^{p}= & a_{q}^{p} \Gamma_{k l}^{q} \tilde{a}_{i}^{k} \tilde{a}_{j}^{l}+a_{q r}^{p} \Gamma_{k}^{q} \Gamma_{l}^{r} \tilde{a}_{i}^{k} \tilde{a}_{j}^{l}+a_{q k}^{p} \Gamma_{l}^{q} \tilde{a}_{i}^{k} \tilde{a}_{j}^{l}+a_{q l}^{p} \Gamma_{k}^{q} \tilde{a}_{i}^{k} \tilde{a}_{j}^{l}+a_{q}^{p} \Gamma_{k}^{q} \tilde{a}_{i j}^{k} \\
& +a_{k}^{p} \tilde{a}_{i j}^{k}+a_{k l}^{p} \tilde{a}_{i}^{k} \tilde{a}_{j}^{l} \\
\bar{\Gamma}_{i q}^{p}= & a_{r}^{p} \Gamma_{j s}^{r} \tilde{a}_{q}^{s} \tilde{a}_{i}^{j}+a_{r s}^{p} \Gamma_{j}^{r} \tilde{a}_{q}^{s} \tilde{a}_{i}^{j}+a_{r j}^{p} \tilde{a}_{q}^{r} \tilde{a}_{i}^{j} \\
\bar{\Gamma}_{i j k}^{p}= & \left(a_{q n}^{p}+a_{q r}^{p} \Gamma_{n}^{r}\right) \Gamma_{l m}^{q}+\left(a_{n q r}^{p}+a_{q r s}^{p} \Gamma_{n}^{s}\right) \Gamma_{l}^{q} \Gamma_{m}^{r}+a_{q}^{p} \Gamma_{l m n}^{q} \\
& +a_{q r}^{p}\left(\Gamma_{l n}^{q} \Gamma_{m}^{r}+\Gamma_{l}^{q} \Gamma_{m n}^{r}\right)+\left(a_{q l n}^{p}+a_{q r l}^{p} \Gamma_{n}^{r}\right) \Gamma_{m}^{q}+a_{q l}^{p} \Gamma_{m n}^{q} \\
& \left.+\left(a_{q m n}^{p}+a_{q r m}^{p} \Gamma_{n}^{r}\right) \Gamma_{l}^{q}+a_{q m}^{p} \Gamma_{l n}^{q}+a_{l m n}^{p}+a_{l m q}^{p} \Gamma_{n}^{q}\right] \tilde{a}_{i}^{l} \tilde{a}_{j}^{m} \tilde{a}_{k}^{n} \\
& +\left(a_{q}^{p} \Gamma_{l m}^{q}+a_{q r}^{p} \Gamma_{l}^{q} \Gamma_{m}^{r}+a_{q l}^{p} \Gamma_{m}^{q}+a_{q m}^{p} \Gamma_{l}^{q}+a_{l m}^{p}\right)\left(\tilde{a}_{i k}^{l} \tilde{a}_{j}^{m}+\tilde{a}_{i}^{l} \tilde{a}_{j k}^{m}\right) \\
& +\left[\left(a_{q n}^{p}+a_{q r}^{p} \Gamma_{n}^{r}\right) \Gamma_{l}^{q}+a_{q}^{p} \Gamma_{l n}^{q}+a_{l n}^{p}+a_{q l}^{p} \Gamma_{n}^{q}\right] \tilde{a}_{i j}^{l} \tilde{a}_{k}^{n}+\left(a_{q}^{p} \Gamma_{l}^{q}+a_{l}^{p}\right) \tilde{a}_{i j k}^{l} \\
\bar{\Gamma}_{i q r}^{p}= & \left(a_{s u}^{p} \Gamma_{j t}^{s}+a_{s}^{p} \Gamma_{j t u}^{s}+a_{s t u}^{p} \Gamma_{j}^{s}+a_{s t}^{p} \Gamma_{j u}^{s}+a_{j t u}^{p}\right) \tilde{a}_{i}^{j} \tilde{a}_{q}^{t} \tilde{a}_{r}^{u} \\
\bar{\Gamma}_{i j q}^{p}= & \left(a_{r t}^{p} \Gamma_{k l}^{r}+a_{r}^{p} \Gamma_{k l t}^{r}+a_{r s t}^{p} \Gamma_{k}^{r} \Gamma_{l}^{s}+a_{r s}^{p} \Gamma_{k t}^{r} \Gamma_{l}^{s}+a_{r s}^{p} \Gamma_{k}^{r} \Gamma_{l t}^{s}+a_{r k t}^{p} \Gamma_{l}^{r}\right. \\
& \left.+a_{r k}^{p} \Gamma_{l t}^{r}+a_{r l t}^{p} \Gamma_{k}^{r}+a_{r l}^{p} \Gamma_{k t}^{r}+a_{k l t}^{p}\right) \tilde{a}_{i}^{k} \tilde{a}_{j}^{l} \tilde{a}_{q}^{t} \\
& +\left(a_{r t}^{p} \Gamma_{k}^{r}+a_{r}^{p} \Gamma_{k t}^{r}+a_{k t}^{p}\right) \tilde{a}_{i j}^{k} \tilde{a}_{q}^{t} .
\end{aligned}
$$

The action on $\Lambda$ is

$$
\begin{aligned}
\bar{\nabla}_{j k}^{i}= & a_{l}^{i} \nabla_{m n}^{l} \tilde{a}_{j}^{m} \tilde{a}_{k}^{n}+a_{l m}^{i} \tilde{a}_{j}^{l} \tilde{a}_{k}^{m} \\
\bar{\nabla}_{j k l}^{i}= & a_{p}^{i} \nabla_{m n q}^{p} \tilde{a}_{l}^{q} \tilde{a}_{k}^{n} \tilde{a}_{j}^{m}+a_{p}^{i} \nabla_{s m}^{p} \tilde{a}_{l}^{m} \tilde{a}_{j k}^{s}+a_{p s}^{i} \nabla_{m n}^{p} \tilde{a}_{l}^{n} \tilde{a}_{j}^{m} \tilde{a}_{k}^{s}+a_{p s}^{i} \nabla_{n m}^{s} \tilde{a}_{l}^{m} \tilde{a}_{j}^{p} \tilde{a}_{k}^{n} \\
& +a_{m n q}^{i} \tilde{a}_{l}^{q} \tilde{a}_{k}^{m} \tilde{a}_{j}^{n}+a_{s m}^{i} \tilde{a}_{k j}^{s} \tilde{a}_{l}^{m} .
\end{aligned}
$$

Finally, the group $G_{m, n}^{3}$ acts on $Z$ in the form

$$
\begin{aligned}
\bar{Z}_{k}^{p}= & a_{q}^{p} Z_{l}^{q} \tilde{a}_{k}^{l} \\
\bar{Z}_{i ; k}^{p}= & a_{q r}^{p} Y^{r} y_{j}^{q} \omega_{l} \tilde{a}_{i}^{j} \tilde{a}_{k}^{l}+a_{q}^{p} Z_{j ; l}^{q} \tilde{a}_{i}^{j} \tilde{a}_{k}^{l}+a_{q j}^{p} Z_{l}^{q} \tilde{a}_{i}^{j} \tilde{a}_{k}^{l} \\
\bar{Z}_{i j ; k}^{p}= & a_{q r}^{p} Y^{r} y_{l m}^{q} \omega_{n} \tilde{a}_{i}^{l} \tilde{a}_{j}^{m} \tilde{a}_{k}^{n}+a_{q}^{p} Z_{l m ; n}^{q} \tilde{a}_{i}^{l} \tilde{a}_{j}^{m} \tilde{a}_{k}^{n}+a_{q r s}^{p} Y^{s} y_{l}^{q} y_{m}^{r} \omega_{n} \tilde{a}_{i}^{l} \tilde{a}_{j}^{m} \tilde{a}_{k}^{n} \\
& +a_{q r}^{p}\left(Y_{l}^{q} y_{m}^{r}+y_{l}^{q} Y_{m}^{r}\right) \omega_{n} \tilde{a}_{i}^{l} \tilde{a}_{j}^{m} \tilde{a}_{k}^{n}+a_{q r l}^{p} Y^{r} y_{m}^{q} \omega_{n} \tilde{a}_{i}^{l} \tilde{a}_{j}^{m} \tilde{a}_{k}^{n} \\
& +a_{q l}^{p} Z_{m ; n}^{q} \tilde{a}_{i}^{l} \tilde{a}_{j}^{m} \tilde{a}_{k}^{n}+a_{q r m}^{p} Y^{r} y_{l}^{q} \omega_{n} \tilde{a}_{i}^{l} \tilde{a}_{j}^{m} \tilde{a}_{k}^{n}+a_{q m}^{p} Z_{l ; n}^{q} \tilde{a}_{i}^{l} \tilde{a}_{j}^{m} \tilde{a}_{k}^{n} \\
& +a_{q r}^{p} Y^{r} y_{l}^{q} \omega_{n} \tilde{a}_{i j}^{l} \tilde{a}_{k}^{n}+a_{q}^{p} Z_{l ; n}^{q} \tilde{a}_{i j}^{l} \tilde{a}_{k}^{n}+a_{q l}^{p} Z_{n}^{q} \tilde{a}_{i j}^{l} \tilde{a}_{k}^{n}+a_{q l m}^{p} Z_{n}^{q} \tilde{a}_{i}^{l} \tilde{a}_{j}^{m} \tilde{a}_{k}^{n} .
\end{aligned}
$$

Now we want to show that every $\mathcal{F} \mathcal{M}_{m, n}$-natural operator $\Delta_{D}: J^{1} \times$ $Q_{\tau}(\mathcal{B}) \rightsquigarrow\left(J^{2}, T^{*} \mathcal{B} \otimes V J^{2}\right)$ is of order 2 in $\Gamma$ and of order 1 in $\nabla$. Using the general theory, the operators in question are in bijection with $G_{m, n}^{q}$-invariant maps

$$
f: J_{0}^{r}\left(J^{1} \mathbf{R}^{m, n}\right) \times J_{0}^{s}\left(Q_{\tau}\left(\mathbf{R}^{m}\right)\right) \times J_{0}^{2} \mathbf{R}^{m, n} \rightarrow\left(T^{*} \mathbf{R}^{m} \otimes V J^{2} \mathbf{R}^{m, n}\right)_{0},
$$


where $q=\max \left\{\operatorname{rank}\left(J^{r} J^{1}\right), \operatorname{rank}\left(J^{s} Q_{\tau}\right), \operatorname{rank}\left(J^{2}\right), \operatorname{rank}\left(T^{*}\right), \operatorname{rank}\left(V J^{2}\right)\right\}=$ $\max \{r+1, s+2,2,1,3\}=\max \{r+1, s+2,3\} \geq 3$.

We shall investigate these maps. Let $\alpha$ and $\gamma$ be multi indices in $x^{i}$ and $\beta$ be a multi index in $y^{p}$. This associated map of our operator has the form

$$
\begin{aligned}
Z_{k}^{p} & =f_{k}^{p}\left(\left(\Gamma_{i}^{p}\right)_{\alpha \beta},\left(\nabla_{j k}^{i}\right)_{\gamma}, y_{i}^{p}, y_{i j}^{p}\right) \\
Z_{i ; k}^{p} & =f_{i ; k}^{p}\left(\left(\Gamma_{i}^{p}\right)_{\alpha \beta},\left(\nabla_{j k}^{i}\right)_{\gamma}, y_{i}^{p}, y_{i j}^{p}\right) \\
Z_{i j ; k}^{p} & =f_{i j ; k}^{p}\left(\left(\Gamma_{i}^{p}\right)_{\alpha \beta},\left(\nabla_{j k}^{i}\right)_{\gamma}, y_{i}^{p}, y_{i j}^{p}\right),
\end{aligned}
$$

where $|\alpha|+|\beta| \leq r$ and $|\gamma| \leq s$.

Using the homotheties

$$
\begin{aligned}
& \tilde{a}_{j}^{i}=t \delta_{j}^{i}, \tilde{a}_{q}^{p}=\delta_{q}^{p}, a_{i}^{p}=0, a_{q r}^{p}=0, a_{q i}^{p}=0, \tilde{a}_{i j}^{k}=0, a_{i j}^{p}=0, a_{q r i}^{p}=0, \\
& a_{q r s}^{p}=0, a_{q i j}^{p}=0, a_{i j k}^{p}=0, \tilde{a}_{i j k}^{l}=0,
\end{aligned}
$$

we obtain

$$
t f_{k}^{p}=f_{k}^{p}\left(t^{1+|\alpha|}\left(\Gamma_{i}^{p}\right)_{\alpha \beta}, t^{1+|\gamma|}\left(\nabla_{j k}^{i}\right)_{\gamma}, t y_{i}^{p}, t^{2} y_{i j}^{p}\right) .
$$

From the homogeneous function theorem we deduce that $f_{k}^{p}$ is linear in $\left(\Gamma_{i}^{p}\right)_{\beta}, \nabla_{j k}^{i}, y_{i}^{p}$ and is independent of $y_{i j}^{p}$ and of the variables with $|\alpha|>0$ or $|\gamma|>0$. Therefore,

$$
f_{k}^{p}=f_{k}^{p}\left(\left(\Gamma_{i}^{p}\right)_{\beta}, \nabla_{j k}^{i}, y_{i}^{p}\right)
$$

Considering invariance of (12) with respect to the homotheties

$$
\begin{aligned}
& \tilde{a}_{j}^{i}=\delta_{j}^{i}, a_{q}^{p}=t \delta_{q}^{p}, a_{i}^{p}=0, a_{q r}^{p}=0, a_{q i}^{p}=0, \tilde{a}_{i j}^{k}=0, a_{i j}^{p}=0, a_{q r i}^{p}=0, \\
& a_{q r s}^{p}=0, a_{q i j}^{p}=0, a_{i j k}^{p}=0, \tilde{a}_{i j k}^{l}=0,
\end{aligned}
$$

we get the condition

$$
t f_{k}^{p}=f_{k}^{p}\left(t^{1-|\beta|}\left(\Gamma_{i}^{p}\right)_{\beta}, \nabla_{j k}^{i}, t y_{i}^{p}\right) .
$$

Using again the homogeneous function theorem, we see that $f_{k}^{p}$ is independent of $\left(\Gamma_{i}^{p}\right)_{\beta}$ with $|\beta|>1$.

For $f_{i ; k}^{p}$, the homotheties

$$
\begin{aligned}
& \tilde{a}_{j}^{i}=t \delta_{j}^{i}, \tilde{a}_{q}^{p}=\delta_{q}^{p}, a_{i}^{p}=0, a_{q r}^{p}=0, a_{q i}^{p}=0, \tilde{a}_{i j}^{k}=0, a_{i j}^{p}=0, a_{q r i}^{p}=0, \\
& a_{q r s}^{p}=0, a_{q i j}^{p}=0, a_{i j k}^{p}=0, \tilde{a}_{i j k}^{l}=0,
\end{aligned}
$$

yield

$$
t^{2} f_{i ; k}^{p}=f_{i ; k}^{p}\left(t^{1+|\alpha|}\left(\Gamma_{i}^{p}\right)_{\alpha \beta}, t^{1+|\gamma|}\left(\nabla_{j k}^{i}\right)_{\gamma}, t y_{i}^{p}, t^{2} y_{i j}^{p}\right)
$$

so that $f_{i ; k}^{p}$ is a polynomial independent of the variables with $|\alpha|>1$ or $|\gamma|>1$. In other words,

$$
f_{i ; k}^{p}=f_{i ; k}^{p}\left(\left(\Gamma_{i}^{p}\right)_{\alpha \beta},\left(\nabla_{j k}^{i}\right)_{\gamma}, y_{i}^{p}, y_{i j}^{p}\right)
$$

for $|\alpha| \leq 1$ and $|\gamma| \leq 1$. 
The homotheties

$$
\begin{aligned}
& \tilde{a}_{j}^{i}=\delta_{j}^{i}, a_{q}^{p}=t \delta_{q}^{p}, a_{i}^{p}=0, a_{q r}^{p}=0, a_{q i}^{p}=0, \tilde{a}_{i j}^{k}=0, a_{i j}^{p}=0, a_{q r i}^{p}=0, \\
& a_{q r s}^{p}=0, a_{q i j}^{p}=0, a_{i j k}^{p}=0, \tilde{a}_{i j k}^{l}=0,
\end{aligned}
$$

imply

$$
t f_{i ; k}^{p}=f_{i ; k}^{p}\left(t^{1-|\beta|}\left(\Gamma_{i}^{p}\right)_{\alpha \beta},\left(\nabla_{j k}^{i}\right)_{\gamma}, t y_{i}^{p}, t y_{i j}^{p}\right)
$$

for $|\alpha| \leq 1$ and $|\gamma| \leq 1$. Therefore we deduce that $f_{i ; k}^{p}$ is independent of $\left(\Gamma_{i}^{p}\right)_{\alpha \beta}$ for $|\alpha|+|\beta|>2$ and $\left(\nabla_{j k}^{i}\right)_{\gamma}$ for $|\gamma|>1$.

Now invariance of $f_{i j ; k}^{p}$ with respect to the homotheties

$$
\begin{aligned}
& \tilde{a}_{j}^{i}=t \delta_{j}^{i}, \tilde{a}_{q}^{p}=t \delta_{q}^{p}, a_{i}^{p}=0, a_{q r}^{p}=0, a_{q i}^{p}=0, \tilde{a}_{i j}^{k}=0, a_{i j}^{p}=0, a_{q r i}^{p}=0, \\
& a_{q r s}^{p}=0, a_{q i j}^{p}=0, a_{i j k}^{p}=0, \tilde{a}_{i j k}^{l}=0,
\end{aligned}
$$

gives

$$
t^{2} f_{i j ; k}^{p}=f_{i j ; k}^{p}\left(t^{|\alpha|+|\beta|}\left(\Gamma_{i}^{p}\right)_{\alpha \beta}, t^{1+|\gamma|}\left(\nabla_{j k}^{i}\right)_{\gamma}, y_{i}^{p}, t y_{i j}^{p}\right) .
$$

So $f_{i j ; k}^{p}$ is a polynomial independent of $\left(\Gamma_{i}^{p}\right)_{\alpha \beta}$ for $|\alpha|+|\beta|>2$ and $\left(\nabla_{j k}^{i}\right)_{\gamma}$ for $|\gamma|>1$. Hence the associated map of our operator is independent of $\left(\Gamma_{i}^{p}\right)_{\alpha \beta}$ for $|\alpha|+|\beta|>2$ and $\left(\nabla_{j k}^{i}\right)_{\gamma}$ for $|\gamma|>1$. This completes the proof of the fact that $\mathcal{F} \mathcal{M}_{m, n}$-natural operator $\Delta_{D}: J^{1} \times Q_{\tau}(\mathcal{B}) \rightsquigarrow\left(J^{2}, T^{*} \mathcal{B} \otimes V J^{2}\right)$ is of order 2 in $\Gamma$ and of order 1 in $\nabla$. In other words it means that the value $\Delta_{D}(\Gamma, \nabla)(\rho)$ is determined by $j_{(0,0)}^{2} \Gamma$ and $j_{0}^{1}(\nabla)$ and $\rho$ for any $\Gamma \in \operatorname{Con}\left(\mathbf{R}^{m, n}\right), \nabla \in Q_{\tau}\left(\mathbf{R}^{m}\right)$ and $\rho \in\left(J^{2} \mathbf{R}^{m, n}\right)_{(0,0)}$.

In the rest of the proof, we shall use $\left(\Gamma, \nabla, y_{0}, 3\right)$-quasi-normal fibred coordinate systems,only. Consider the case $m \geq 2$.

Since $\Delta_{D}$ is invariant with respect to $\left(\Gamma, \nabla, y_{0}, 3\right)$-quasi-normal fibred coordinate systems, $\Delta_{D}$ is determined by the contractions $\left\langle\Delta_{D}(\Gamma, \nabla)(\rho), v\right\rangle \in$ $V_{\rho} J^{2} \mathbf{R}^{m, n}$ for all $\rho \in\left(J^{2} \mathbf{R}^{m, n}\right)_{(0,0)}$, all $v \in T_{0} \mathbf{R}^{m}$, all general connections $\Gamma$ on $\mathbf{R}^{m, n}$ and all torsion free classical linear connections $\nabla$ on $\mathbf{R}^{m}$ such that $\psi=i d_{\mathbf{R}^{m, n}}$ is a $(\Gamma, \nabla,(0,0), 3)$-quasi-normal fibred coordinate system on $\mathbf{R}^{m, n}$ over $\psi=i d_{\mathbf{R}^{m}}$.

For vector bundles $E \rightarrow M$ we have the standard identification $V E=$ $E \times{ }_{M} E$ which is a vector bundle isomorphism. As $\mathbf{R}^{m, n}$ is a vector bundle and $J^{2} \mathbf{R}^{m, n}$ is a vector bundle we can write that $V_{\rho} J^{2} \mathbf{R}^{m, n} \cong{ }_{\rho} J_{0}^{2} \mathbf{R}^{m, n}$. This identification $\cong_{\rho}$ is $G L(m) \times G L(n)$-invariant but not $\mathcal{F} \mathcal{M}_{m, n}$-invariant.

Next we use the usual $G L(m) \times G L(n)$-invariant identification

$$
J_{0}^{2} \mathbf{R}^{m, n} \cong \oplus_{k=0}^{2} S^{k} \mathbf{R}^{m *} \otimes \mathbf{R}^{n}
$$

(it is not $\mathcal{F} \mathcal{M}_{m, n}$-invariant). Therefore, the values $\left\langle\Delta_{D}(\Gamma, \nabla)(\rho), v\right\rangle$ are determined by the values $\psi_{\Gamma, \nabla}^{k}(\rho, v) \in S^{k} \mathbf{R}^{m *} \otimes \mathbf{R}^{n}$ for $k=0,1,2$ obtained by composing the values $\left\langle\Delta_{D}(\Gamma, \nabla)(\rho), v\right\rangle$ with the respective projections. So we can write

$$
\left\langle\Delta_{D}(\Gamma, \nabla)(\rho), v\right\rangle \cong \psi_{\Gamma, \nabla}^{0}(\rho, v) \oplus \psi_{\Gamma, \nabla}^{1}(\rho, v) \oplus \psi_{\Gamma, \nabla}^{2}(\rho, v),
$$


where $\psi_{\Gamma, \nabla}^{0}(\rho, v) \in \mathbf{R}^{n}, \psi_{\Gamma, \nabla}^{1}(\rho, v) \in \mathbf{R}^{m *} \otimes \mathbf{R}^{n}, \psi_{\Gamma, \nabla}^{2}(\rho, v) \in S^{2} \mathbf{R}^{m *} \otimes \mathbf{R}^{n}$.

Now the values $\psi_{\Gamma, \nabla}^{k}(\rho, v) \in S^{k} \mathbf{R}^{m *} \otimes \mathbf{R}^{n}$ for $k=0,1$ are determined by the contractions $\left\langle\psi_{\Gamma, \nabla}^{0}(\rho, v), u\right\rangle,\left\langle\psi_{\Gamma, \nabla}^{1}(\rho, v), w \otimes u\right\rangle$ for all $v \in T_{0} \mathbf{R}^{m} \cong \mathbf{R}^{m}$, $u \in \mathbf{R}^{n *}, w \in \mathbf{R}^{m}$ and all $\Gamma, \nabla$ in question.

Using the polarization formula from linear algebra, we have that every symmetric bilinear form on a vector space is uniquely determined by the corresponding quadratic form. Therefore, for $k=2$ the values $\psi_{\Gamma, \nabla}^{2}(\rho, v)$ are determined by the contractions $\left\langle\psi_{\Gamma, \nabla}^{2}(\rho, v),(w \odot w) \otimes u\right\rangle$ for all $v, u, w, \Gamma, \nabla$ as above, where $\odot$ denotes the symmetric tensor product. Then by the density argument and $m \geq 2$, we can assume that $v$ and $w$ are linearly independent and $u \neq 0$.

Using the $G L(m) \times G L(n)$-invariance of $\Delta_{D}$ and Proposition 1 , we can assume $v=e_{1}, w=e_{2}, u=E^{1}$, where $\left(e_{i}\right)$ is the standard basis in $\mathbf{R}^{m},\left(E_{p}\right)$ is the standard basis in $\mathbf{R}^{n}$ and $\left(E^{p}\right)$ is the dual basis in $\mathbf{R}^{n *}$. So we get that the operator $\Delta_{D}$ is uniquely determined by the values $\left\langle\psi_{\Gamma, \nabla}^{0}\left(\rho,\left.\frac{\partial}{\partial x^{1}}\right|_{0}\right), E^{1}\right\rangle$, $\left\langle\psi_{\Gamma, \nabla}^{1}\left(\rho,\left.\frac{\partial}{\partial x^{1}}\right|_{0}\right), e_{2} \otimes E^{1}\right\rangle$ and $\left\langle\psi_{\Gamma, \nabla}^{2}\left(\rho,\left.\frac{\partial}{\partial x^{1}}\right|_{0}\right),\left(e_{2} \odot e_{2}\right) \otimes E^{1}\right\rangle$. In other words, $\Delta_{D}$ is uniquely determined by the values

$$
\begin{array}{r}
\left\langle Y_{\mid \rho}^{1},\left\langle\Delta_{D}(\Gamma, \nabla)(\rho),\left.\frac{\partial}{\partial x^{1}}\right|_{0}\right\rangle\right\rangle \in \mathbf{R} \\
\left\langle Y_{2 \mid \rho}^{1},\left\langle\Delta_{D}(\Gamma, \nabla)(\rho),\left.\frac{\partial}{\partial x^{1}}\right|_{0}\right\rangle\right\rangle \in \mathbf{R} \\
\left\langle Y_{22 \mid \rho}^{1},\left\langle\Delta_{D}(\Gamma, \nabla)(\rho),\left.\frac{\partial}{\partial x^{1}}\right|_{0}\right\rangle\right\rangle \in \mathbf{R}
\end{array}
$$

for all $\rho \in\left(J^{2} \mathbf{R}^{m, n}\right)_{(0,0)}$, all general connections $\Gamma$ on $\mathbf{R}^{m, n}$ and all torsion free classical linear connections $\nabla$ on $\mathbf{R}^{m}$ such that $\psi=i d_{\mathbf{R}^{m, n}}$ is a $(\Gamma, \nabla,(0,0), 3)$-quasi-normal fibred coordinate system on $\mathbf{R}^{m, n}$ over $\underline{\psi}=$ $i d_{\mathbf{R}^{m}}$.

Consider locally defined $\mathcal{F} \mathcal{M}_{m, n}$-maps $\psi_{2}: \mathbf{R}^{m, n} \rightarrow \mathbf{R}^{m, n}, \psi_{3}: \mathbf{R}^{m, n} \rightarrow$ $\mathbf{R}^{m, n}$ given by

$$
\begin{aligned}
& \psi_{2}(x, y)=\left(x, y_{1}+\left(y_{1}\right)^{2}, y_{2}, \ldots, y_{n}\right) \\
& \psi_{3}(x, y)=\left(x, y_{1}+\left(y_{1}\right)^{3}, y_{2}, \ldots, y_{n}\right)
\end{aligned}
$$

for $x \in \mathbf{R}^{n}$ and $y=\left(y_{1}, y_{2}, \ldots, y_{n}\right) \in \mathbf{R}^{n}$. They preserve $\left.\frac{\partial}{\partial x^{1}}\right|_{0}$ and can be written in the form $\psi_{a}(x, y)=\left(i d_{\mathbf{R}^{m}}(x), H_{a}(y)\right)$, where $H_{a}(y)=\left(y_{1}+\right.$ $\left.\left(y_{1}\right)^{a}, y_{2}, \ldots, y_{n}\right)$ and $a=2,3$. So $\psi_{a}=i d_{\mathbf{R}^{m}} \times H_{a}$ for $H_{a}: \mathbf{R}^{n} \rightarrow \mathbf{R}^{n}$ being a diffeomorphism preserving 0 . Hence by Proposition 1 these $\mathcal{F} \mathcal{M}_{m, n}$-maps $\psi_{a}: \mathbf{R}^{m, n} \rightarrow \mathbf{R}^{m, n}$ for $a=2,3$ transform quasi-normal fibred coordinate systems into quasi-normal ones. Using the invariance of $\Delta_{D}$ with respect to $\psi_{a}: \mathbf{R}^{m, n} \rightarrow \mathbf{R}^{m, n}$ for $a=2,3$ and the density argument, we show that the values $\left\langle Y_{2 \mid \rho}^{1},\left\langle\Delta_{D}(\Gamma, \nabla)(\rho),\left.\frac{\partial}{\partial x^{1}}\right|_{0}\right\rangle\right\rangle$ and $\left\langle Y_{\mid \rho}^{1},\left\langle\Delta_{D}(\Gamma, \nabla)(\rho),\left.\frac{\partial}{\partial x^{1}}\right|_{0}\right\rangle\right\rangle$ 
for all $\Gamma \in \operatorname{Con}\left(\mathbf{R}^{m, n}\right), \nabla \in Q_{\tau}\left(\mathbf{R}^{m}\right), \rho \in\left(J^{2} \mathbf{R}^{m, n}\right)_{(0,0)}$ are determined by the values $\left\langle Y_{22 \mid \rho}^{1},\left\langle\Delta_{D}(\Gamma, \nabla)(\rho),\left.\frac{\partial}{\partial x^{1}}\right|_{0}\right\rangle\right\rangle$ for all $\Gamma, \nabla, \rho$ as above.

Using the action of the group $G_{m, n}^{3}$ on $S_{0}$ for $a=2$, we obtain $\bar{y}_{22}^{1}=$ $y_{22}^{1}+2 y^{1} y_{22}^{1}+2\left(y_{2}^{1}\right)^{2}$ and then

(15) $\bar{Y}_{22}^{1}=d \bar{y}_{22}^{1}=Y_{22}^{1}+4 y_{2}^{1} Y_{2}^{1}+2 y_{22}^{1} Y^{1}+2 y^{1} Y_{22}^{1}=Y_{22}^{1}+4 y_{2}^{1} Y_{2}^{1}+2 y_{22}^{1} Y^{1}$

over $(0,0) \in \mathbf{R}^{m, n}$ (i.e. for $y^{1}=0$ ). Similarly, for $a=3$ we get $\tilde{y}_{22}^{1}=$ $y_{22}^{1}+3\left(y^{1}\right)^{2} y_{22}^{1}+6 y^{1}\left(y_{2}^{1}\right)^{2}$ and then

$$
\begin{aligned}
\tilde{Y}_{22}^{1} & =d \tilde{y}_{22}^{1}=Y_{22}^{1}+6\left(y_{2}^{1}\right)^{2} Y^{1}+6 y^{1} y_{22}^{1} Y^{1}+3\left(y^{1}\right)^{2} Y_{22}^{1}+12 y^{1} y_{2}^{1} Y_{2}^{1} \\
& =Y_{22}^{1}+6\left(y_{2}^{1}\right)^{2} Y^{1}
\end{aligned}
$$

over $(0,0) \in \mathbf{R}^{m, n}$

By formula (16) for $y_{2}^{1}(\rho) \neq 0$, we have

$$
Y^{1}=\frac{\tilde{Y}_{22}^{1}-Y_{22}^{1}}{6\left(y_{2}^{1}\right)^{2}}
$$

and consequently the values $\left\langle Y_{\mid \rho}^{1},\left\langle\Delta_{D}(\Gamma, \nabla)(\rho),\left.\frac{\partial}{\partial x^{1}}\right|_{0}\right\rangle\right\rangle$ for all $\Gamma \in$ $\operatorname{Con}\left(\mathbf{R}^{m, n}\right), \nabla \in Q_{\tau}\left(\mathbf{R}^{m}\right), \rho \in\left(J^{2} \mathbf{R}^{m, n}\right)_{(0,0)}$ are determined by the values $\left\langle Y_{22 \mid \rho}^{1},\left\langle\Delta_{D}(\Gamma, \nabla)(\rho),\left.\frac{\partial}{\partial x^{1}}\right|_{0}\right\rangle\right\rangle$ for all $\Gamma, \nabla, \rho$ as above.

Then analogously from (15) and (17), we see that

$$
Y_{2}^{1}=\frac{\left(\bar{Y}_{22}^{1}-Y_{22}^{1}\right) \cdot 3\left(y_{2}^{1}\right)^{2}-y_{22}^{1}\left(\tilde{Y}_{22}^{1}-Y_{22}^{1}\right)}{12\left(y_{2}^{1}\right)^{3}}
$$

and therefore, the values $\left\langle Y_{2 \mid \rho}^{1},\left\langle\Delta_{D}(\Gamma, \nabla)(\rho),\left.\frac{\partial}{\partial x^{1}}\right|_{0}\right\rangle\right\rangle$ for all $\Gamma \in \operatorname{Con}\left(\mathbf{R}^{m, n}\right)$, $\nabla \in Q_{\tau}\left(\mathbf{R}^{m}\right), \quad \rho \in\left(J^{2} \mathbf{R}^{m, n}\right)_{(0,0)}$ are determined by the values $\left\langle Y_{22 \mid \rho}^{1},\left\langle\Delta_{D}(\Gamma, \nabla)(\rho), \frac{\partial}{\partial x^{1}} \mid 0\right\rangle\right\rangle$ for all $\Gamma, \nabla, \rho$ as above.

Summing up, we obtain that the operator $\Delta_{D}$ is uniquely determined by the values

$$
\left\langle Y_{22 \mid \rho}^{1},\left\langle\Delta_{D}(\Gamma, \nabla)(\rho),\left.\frac{\partial}{\partial x^{1}}\right|_{0}\right\rangle\right\rangle \in \mathbf{R}
$$

for all general connections $\Gamma$ on $\mathbf{R}^{m, n}$ such that

$$
\begin{aligned}
j_{(0,0)}^{2} \Gamma & =j_{(0,0)}^{2}\left(\Gamma_{0}+\sum_{i, j, k=1}^{m} \sum_{p=1}^{n} a_{k i j}^{p} x^{k} x^{i} d x^{j} \otimes \frac{\partial}{\partial y^{p}}\right. \\
& \left.+\sum_{i, j=1}^{m} \sum_{p, q=1}^{n} b_{q i j}^{p} y^{q} x^{i} d x^{j} \otimes \frac{\partial}{\partial y^{p}}+\sum_{i, j=1}^{m} \sum_{p=1}^{n} c_{i j}^{p} x^{i} d x^{j} \otimes \frac{\partial}{\partial y^{p}}\right)
\end{aligned}
$$

for unique real numbers $a_{k i j}^{p}, b_{q i j}^{p}$ and $c_{i j}^{p}$ satisfying (2) and all torsion free classical linear connections $\nabla$ such that the identity map $i d_{\mathbf{R}^{m}}$ is a $\nabla$-normal coordinate system with center zero (then $j_{0}^{1}(\nabla)=j_{0}^{1}\left(\left(\sum_{k=1}^{m} \nabla_{i j ; k}^{l} x^{k}\right)_{i, j, l=1}^{m}\right)$ 
for some $\nabla_{i j ; k}^{l}=\nabla_{j i ; k}^{l} \in \mathbf{R}$ satisfying some "classical" conditions) and all $\rho \in\left(J^{2} \mathbf{R}^{m, n}\right)_{(0,0)}$ of the form

$$
\rho=j_{0}^{2}\left(\left(\sum_{i=1}^{m} g_{i}^{p} x^{i}+\sum_{i, j=1}^{m} h_{i j}^{p} x^{i} x^{j}\right)_{p=1}^{n}\right)
$$

for real numbers $g_{i}^{p}, h_{i j}^{p}=h_{j i}^{p}$. So, it is sufficient to study the values (18) for $\Gamma, \nabla, \rho$ as above.

Equivalently, in terms of $G_{m, n}^{3}$-invariant maps between the standard fibres we obtain that values of functions $f_{1}^{1}$ and $f_{2 ; 1}^{1}$ are determined by values of functions $f_{22 ; 1}^{1}$. So we will study the values

$$
\begin{aligned}
f_{22 ; 1}^{1}\left(\Gamma_{k i j}^{p}\right. & =a_{k i j}^{p}, \Gamma_{q i j}^{p}=b_{q i j}^{p}, \Gamma_{i j}^{p}=c_{i j}^{p}, \nabla_{i j k}^{l}=\nabla_{i j ; k}^{l}, \\
y_{i}^{p} & \left.=g_{i}^{p}, y_{i j}^{p}=h_{i j}^{p}\right) .
\end{aligned}
$$

The invariance of $f_{i j ; k}^{p}$ with respect to the homotheties

$$
\begin{aligned}
& \tilde{a}_{j}^{i}=t \delta_{j}^{i}, \tilde{a}_{q}^{p}=t \delta_{q}^{p}, a_{i}^{p}=0, a_{q r}^{p}=0, a_{q i}^{p}=0, \tilde{a}_{i j}^{k}=0, a_{i j}^{p}=0, \\
& a_{q r i}^{p}=0, a_{q r s}^{p}=0, a_{q i j}^{p}=0, a_{i j k}^{p}=0, \tilde{a}_{i j k}^{l}=0,
\end{aligned}
$$

yields

$$
t^{2} f_{i j ; k}^{p}=f_{i j ; k}^{p}\left(t^{2} a_{k i j}^{p}, t^{2} b_{q i j}^{p}, t c_{i j}^{p}, t^{2} \nabla_{i j ; k}^{l}, g_{i}^{p}, t h_{i j}^{p}\right)
$$

Then the homogeneous function theorem implies that $f_{i j ; k}^{p}$ is linear in $a_{k i j}^{p}$, $b_{q i j}^{p}, \nabla_{i j ; k}^{l}$, bilinear in $c_{i j}^{p}, h_{i j}^{p}$, quadratic in $c_{i j}^{p}$ and $h_{i j}^{p}$. In other words $f_{i j ; k}^{p}$ is the linear combination of monomials

$$
a_{k i j}^{p}, b_{q i j}^{p}, \nabla_{i j ; k}^{l}, c_{i j}^{p} h_{i_{1} j_{1}}^{p_{1}}, c_{i j}^{p} c_{i_{1} j_{1}}^{p_{1}}, h_{i j}^{p} h_{i_{1} j_{1}}^{p_{1}}
$$

with the coefficients being smooth functions in the coefficients $g_{i}^{p}$ of $\rho$.

Then using the invariance of $f_{i j ; k}^{p}$ with respect to the homotheties

$$
\begin{aligned}
& \tilde{a}_{j}^{i}=\delta_{j}^{i}, a_{q}^{p}=t \delta_{q}^{p}, a_{i}^{p}=0, a_{q r}^{p}=0, a_{q i}^{p}=0, \tilde{a}_{i j}^{k}=0, a_{i j}^{p}=0, \\
& a_{q r i}^{p}=0, a_{q r s}^{p}=0, a_{q i j}^{p}=0, a_{i j k}^{p}=0, \tilde{a}_{i j k}^{l}=0
\end{aligned}
$$

for $t>0$ and the homogeneous function theorem, we observe that the coefficients on $a_{k i j}^{p}$ are constant, the coefficients on $b_{q i j}^{p}$ and $\nabla_{i j ; k}^{l}$ are linear and the coefficients on other terms from (22) are zero.

Then using the invariance of $f_{i j ; k}^{p}$ with respect to the $\mathcal{F} \mathcal{M}_{m, n}$-maps $\psi_{t, \tau}: \mathbf{R}^{m, n} \rightarrow \mathbf{R}^{m, n}$ given by $\psi_{t, \tau}(x, y)=\left(t^{1} x^{1}, \ldots, t^{m} x^{m}, \tau^{1} y^{1}, \ldots, \tau^{n} y^{n}\right)$ 
for $t^{i}>0, i=1, \ldots, m$ and $\tau^{p}>0, p=1, \ldots, n$ we deduce that

$$
\begin{aligned}
f_{22 ; 1}^{1} & =\left(\alpha_{1} a_{122}^{1}+\alpha_{2} a_{212}^{1}+\alpha_{3} a_{221}^{1}\right) \\
& +\left(\sum_{q=1}^{n} \beta_{q 12} b_{q 12}^{1} g_{2}^{q}+\sum_{q=1}^{n} \beta_{q 21} b_{q 21}^{1} g_{2}^{q}+\sum_{q=1}^{n} \beta_{q 22} b_{q 22}^{1} g_{1}^{q}\right) \\
& +\left(\sum_{q=1}^{n} \gamma_{q 12} b_{q 12}^{q} g_{2}^{1}+\sum_{q=1}^{n} \gamma_{q 21} b_{q 21}^{q} g_{2}^{1}+\sum_{q=1}^{n} \gamma_{q 22} b_{q 22}^{q} g_{1}^{1}\right)+g\left(\left(g_{l}^{1}\right),\left(\nabla_{i j ; k}^{l}\right)\right)
\end{aligned}
$$

for some uniquely determined real numbers $\alpha_{1}, \alpha_{2}, \alpha_{3}, \beta_{q 12}, \beta_{q 21}, \beta_{q 22}, \gamma_{q 12}$, $\gamma_{q 21}, \gamma_{q 22}$ and some uniquely determined bilinear function $g$.

Now because of conditions (2) we have

$$
\begin{aligned}
f_{22 ; 1}^{1}= & a_{122}^{1}\left(\alpha_{1}+\alpha_{2}-2 \alpha_{3}\right)+\sum_{q=1}^{n}\left(\beta_{q 12}-\beta_{q 21}\right) b_{q 12}^{1} g_{2}^{q} \\
& +\sum_{q=1}^{n}\left(\gamma_{q 12}-\gamma_{q 21}\right) b_{q 12}^{q} g_{2}^{1}+g\left(\left(g_{l}^{1}\right),\left(\nabla_{i j ; k}^{l}\right)\right) \\
= & \alpha a_{122}^{1}+\sum_{q=1}^{n} \beta_{q} b_{q 12}^{1} g_{2}^{q}+\sum_{q=1}^{n} \gamma_{q} b_{q 12}^{q} g_{2}^{1}+g\left(\left(g_{l}^{1}\right),\left(\nabla_{i j ; k}^{l}\right)\right),
\end{aligned}
$$

where $\alpha=\alpha_{1}+\alpha_{2}-2 \alpha_{3}, \beta_{q}=\beta_{q 12}-\beta_{q 21}, \gamma_{q}=\gamma_{q 12}-\gamma_{q 21}$ for $q=1, \ldots, n$. Further evaluations give

$$
\begin{aligned}
f_{22 ; 1}^{1}= & \alpha a_{122}^{1}+\left(\beta_{1}+\gamma_{1}\right) b_{112}^{1} g_{2}^{1}+\sum_{q=2}^{n} \beta_{q} b_{q 12}^{1} g_{2}^{q} \\
& +\sum_{q=2}^{n} \gamma_{q} b_{q 12}^{q} g_{2}^{1}+g\left(\left(g_{l}^{1}\right),\left(\nabla_{i j ; k}^{l}\right)\right) \\
= & \alpha a_{122}^{1}+\beta b_{112}^{1} g_{2}^{1}+\sum_{q=2}^{n} \beta_{q} b_{q 12}^{1} g_{2}^{q}+\sum_{q=2}^{n} \gamma_{q} b_{q 12}^{q} g_{2}^{1}+g\left(\left(g_{l}^{1}\right),\left(\nabla_{i j ; k}^{l}\right)\right),
\end{aligned}
$$

where $\beta=\beta_{1}+\gamma_{1}$. In other words,

$$
\begin{aligned}
\left\langle Y_{22 \mid \rho}^{1},\left\langle\Delta_{D}(\Gamma, \nabla)(\rho),\left.\frac{\partial}{\partial x^{1}}\right|_{0}\right\rangle\right\rangle= & \alpha a_{122}^{1}+\beta b_{112}^{1} g_{2}^{1}+\sum_{q=2}^{n} \beta_{q} b_{q 12}^{1} g_{2}^{q} \\
& +\sum_{q=2}^{n} \gamma_{q} b_{q 12}^{q} g_{2}^{1}+g\left(\left(g_{l}^{1}\right),\left(\nabla_{i j ; k}^{l}\right)\right),
\end{aligned}
$$

for some uniquely determined real numbers $\alpha, \beta, \beta_{q}, \gamma_{q}$ and some uniquely determined bilinear function $g$, where $j_{(0,0)}^{2} \Gamma$ is of the form (19) with the coefficients $a_{k i j}^{p}, b_{q i j}^{p}$ and $c_{i j}^{p}$ satisfying $(2), j_{0}^{1}(\nabla)=j_{0}^{1}\left(\left(\sum_{k=1}^{m} \nabla_{i j ; k}^{l} x^{k}\right)_{i, j, l=1}^{m}\right)$ 
for some $\nabla_{i j ; k}^{l}=\nabla_{j i ; k}^{l} \in \mathbf{R}$ satisfying some "classical" conditions and $\rho$ is of the form (20) with $g_{i}^{p}, h_{i j}^{p}=h_{j i}^{p}$.

From (23) it follows that $\Delta_{D}$ is determined by the real number $\alpha$, the bilinear map $g$ and the values

$$
\begin{aligned}
& \Delta_{D}\left(\Gamma_{0}+\left(x^{1} d x^{2}-x^{2} d x^{1}\right) \otimes \frac{\partial}{\partial y^{1}}\right. \\
& \left.+\sum_{p, q=1}^{n} b_{q 12}^{p} y^{q}\left(x^{1} d x^{2}-x^{2} d x^{1}\right) \otimes \frac{\partial}{\partial y^{p}}, \nabla^{0}\right)(\rho) \\
& =\Delta_{D}\left(\Gamma_{0}+\left(x^{1} d x^{2}-x^{2} d x^{1}\right)\left(\frac{\partial}{\partial y^{1}}+\sum_{p, q=1}^{n} b_{q 12}^{p} y^{q} \frac{\partial}{\partial y^{p}}\right), \nabla^{0}\right)(\rho)
\end{aligned}
$$

for all $b_{q 12}^{p} \in \mathbf{R}$ and all $\rho \in\left(J^{2} \mathbf{R}^{m, n}\right)_{(0,0)}$, where $\nabla^{0}$ is the usual flat torsion free classical linear connection on $\mathbf{R}^{m}$.

Considering the invariance of $\Delta_{D}$ with respect to the maps $i d_{\mathbf{R}^{m}} \times H$ for diffeomorphisms $H: \mathbf{R}^{n} \rightarrow \mathbf{R}^{n}$ preserving 0, we get that $\sum_{p, q=1}^{n} b_{q 12}^{p} y^{q} \frac{\partial}{\partial y^{p}}$ is near 0 equal to zero modulo some diffeomorphism $H: \mathbf{R}^{n} \rightarrow \mathbf{R}^{n}$ preserving 0 . Hence we have that $\Delta_{D}$ is determined by the real number $\alpha$, the bilinear map $g$ and the values

$$
\Delta_{D}\left(\Gamma_{0}+a\left(x^{1} d x^{2}-x^{2} d x^{1}\right) \otimes \frac{\partial}{\partial y^{1}}, \nabla^{0}\right)(\rho)
$$

for all $a \in \mathbf{R}$ and all $\rho \in\left(J^{2} \mathbf{R}^{m, n}\right)_{(0,0)}$.

Next using the invariance of $\Delta_{D}$ with respect to the homotheties

$$
\begin{aligned}
& \tilde{a}_{j}^{i}=\delta_{j}^{i}, a_{q}^{p}=t \delta_{q}^{p}, a_{i}^{p}=0, a_{q r}^{p}=0, a_{q i}^{p}=0, \tilde{a}_{i j}^{k}=0, a_{i j}^{p}=0, \\
& a_{q r i}^{p}=0, a_{q r s}^{p}=0, a_{q i j}^{p}=0, a_{i j k}^{p}=0, \tilde{a}_{i j k}^{l}=0,
\end{aligned}
$$

from the homogeneous function theorem, it follows that (25) depends linearly in $(a, \rho)$. This implies that $\Delta_{D}$ is determined by the real number $\alpha$, the bilinear map $g$ and the values

$$
\Delta_{D}\left(\Gamma_{0}+\left(x^{1} d x^{2}-x^{2} d x^{1}\right) \otimes \frac{\partial}{\partial y^{1}}, \nabla^{0}\right)\left(j_{0}^{2} 0\right) \text { and } \Delta_{D}\left(\Gamma_{0}, \nabla^{0}\right)(\rho)
$$

for all $\rho \in\left(J^{2} \mathbf{R}^{m, n}\right)_{(0,0)}$.

Now the values $\Delta_{D}\left(\Gamma_{0}, \nabla^{0}\right)(\rho)$ are determined by the values $\left\langle\Delta_{D}\left(\Gamma_{0}, \nabla^{0}\right)(\rho), v\right\rangle \in V_{\rho} J^{2} \mathbf{R}^{m, n} \cong J_{0}^{2} \mathbf{R}^{m, n} \cong \oplus_{k=0}^{2} S^{k} \mathbf{R}^{m *} \otimes \mathbf{R}^{n}$ for all $\rho \in\left(J^{2} \mathbf{R}^{m, n}\right)_{(0,0)}, v \in T_{0} \mathbf{R}^{m}$ such that $\psi=i d_{\mathbf{R}^{m, n}}$ is a $\left(\Gamma_{0}, \nabla^{0},(0,0), 3\right)$ quasi-normal fibred coordinate system on $\mathbf{R}^{m, n}$ over $\psi=i d_{\mathbf{R}^{m}}$. Since the $\mathcal{F} \mathcal{M}_{m, n}$-maps of the form $B \times H$ (in question) preserve the trivial general connection $\Gamma_{0}$ and the flat torsion free classical linear connection $\nabla^{0}$ then we deduce that the values $\Delta_{D}\left(\Gamma_{0}, \nabla^{0}\right)(\rho)$ are determined by the values 
$\left\langle Y_{22 \mid \rho}^{1},\left\langle\Delta_{D}\left(\Gamma_{0}, \nabla^{0}\right)(\rho),\left.\frac{\partial}{\partial x^{1}}\right|_{0}\right\rangle\right\rangle$. But using the formula (23), we see that the last values are equal to zero. Therefore,

$$
\Delta_{D}\left(\Gamma_{0}, \nabla^{0}\right)(\rho)=0
$$

for any $\rho \in\left(J^{2} \mathbf{R}^{m, n}\right)_{(0,0)}$. This gives that $\Delta_{D}$ is determined by the real number $\alpha$, the bilinear map $g$ and the values

$$
\Delta_{D}\left(\Gamma_{0}+\left(x^{1} d x^{2}-x^{2} d x^{1}\right) \otimes \frac{\partial}{\partial y^{1}}, \nabla^{0}\right)\left(j_{0}^{2} 0\right) .
$$

The value (27) is determined by the evaluations

$$
\begin{array}{r}
\left\langle Y_{\mid j_{0}^{2} 0}^{p},\left\langle\Delta_{D}\left(\Gamma_{0}+\left(x^{1} d x^{2}-x^{2} d x^{1}\right) \otimes \frac{\partial}{\partial y^{1}}, \nabla^{0}\right)\left(j_{0}^{2} 0\right),\left.\frac{\partial}{\partial x^{k}}\right|_{0}\right\rangle\right\rangle \\
\left\langle Y_{i \mid j_{0}^{2} 0}^{p},\left\langle\Delta_{D}\left(\Gamma_{0}+\left(x^{1} d x^{2}-x^{2} d x^{1}\right) \otimes \frac{\partial}{\partial y^{1}}, \nabla^{0}\right)\left(j_{0}^{2} 0\right),\left.\frac{\partial}{\partial x^{k}}\right|_{0}\right\rangle\right\rangle \\
\left\langle Y_{i j \mid j_{0}^{2} 0}^{p},\left\langle\Delta_{D}\left(\Gamma_{0}+\left(x^{1} d x^{2}-x^{2} d x^{1}\right) \otimes \frac{\partial}{\partial y^{1}}, \nabla^{0}\right)\left(j_{0}^{2} 0\right),\left.\frac{\partial}{\partial x^{k}}\right|_{0}\right\rangle\right\rangle
\end{array}
$$

for all $p=1, \ldots, n$ and all $i, j, k=1, \ldots, m$.

Since (25) depends linearly on $a$, using the invariance of $\Delta_{D}$ with respect to the homotheties

$$
\begin{aligned}
& \tilde{a}_{j}^{i}=\delta_{j}^{i}, \tilde{a}_{q}^{p}=t \delta_{q}^{p}, a_{i}^{p}=0, a_{q r}^{p}=0, a_{q i}^{p}=0, \tilde{a}_{i j}^{k}=0, a_{i j}^{p}=0, \\
& a_{q r i}^{p}=0, \quad a_{q r s}^{p}=0, a_{q i j}^{p}=0, a_{i j k}^{p}=0, \tilde{a}_{i j k}^{l}=0,
\end{aligned}
$$

we see that

$$
\begin{aligned}
& \left\langle Y_{\mid j_{0}^{2} 0}^{p},\left\langle\Delta_{D}\left(\Gamma_{0}+\left(x^{1} d x^{2}-x^{2} d x^{1}\right) \otimes \frac{\partial}{\partial y^{1}}, \nabla^{0}\right)\left(j_{0}^{2} 0\right),\left.\frac{\partial}{\partial x^{k}}\right|_{0}\right\rangle\right\rangle=0, \\
& \left\langle Y_{i j \mid j_{0}^{2} 0}^{p},\left\langle\Delta_{D}\left(\Gamma_{0}+\left(x^{1} d x^{2}-x^{2} d x^{1}\right) \otimes \frac{\partial}{\partial y^{1}}, \nabla^{0}\right)\left(j_{0}^{2} 0\right),\left.\frac{\partial}{\partial x^{k}}\right|_{0}\right\rangle\right\rangle=0 .
\end{aligned}
$$

Therefore, $\Delta_{D}$ is determined by the evaluations

$$
\left\langle Y_{i \mid j_{0}^{2} 0}^{p},\left\langle\Delta_{D}\left(\Gamma_{0}+\left(x^{1} d x^{2}-x^{2} d x^{1}\right) \otimes \frac{\partial}{\partial y^{1}}, \nabla^{0}\right)\left(j_{0}^{2} 0\right),\left.\frac{\partial}{\partial x^{k}}\right|_{0}\right\rangle\right\rangle .
$$

Then using the invariance of $\Delta_{D}$ with respect to $a_{t}: \mathbf{R}^{m, n} \rightarrow \mathbf{R}^{m, n}$ by $a_{t}(x, y)=\left(x, t y_{1}, y_{2}, \ldots, y_{n}\right)$ for $t>0$, we may assume $p=1$, i.e. $\Delta_{D}$ is determined by the evaluations

$$
\left\langle Y_{i \mid j_{0}^{2} 0}^{1},\left\langle\Delta_{D}\left(\Gamma_{0}+\left(x^{1} d x^{2}-x^{2} d x^{1}\right) \otimes \frac{\partial}{\partial y^{1}}, \nabla^{0}\right)\left(j_{0}^{2} 0\right),\left.\frac{\partial}{\partial x^{k}}\right|_{0}\right\rangle\right\rangle .
$$

Then using the invariance of $\Delta_{D}$ with respect to $b_{t}: \mathbf{R}^{m, n} \rightarrow \mathbf{R}^{m, n}$ by $b_{t}(x, y)=\left(t_{1} x_{1}, \ldots, t_{m} x_{m}, y_{1}, \ldots, y_{n}\right)$, we see that the values $(30)$ are all zero except the values

$$
\left\langle Y_{1 \mid j_{0}^{2} 0}^{1},\left\langle\Delta_{D}\left(\Gamma_{0}+\left(x^{1} d x^{2}-x^{2} d x^{1}\right) \otimes \frac{\partial}{\partial y^{1}}, \nabla^{0}\right)\left(j_{0}^{2} 0\right),\left.\frac{\partial}{\partial x^{2}}\right|_{0}\right\rangle\right\rangle
$$


and

$$
\left\langle Y_{2 \mid j_{0}^{2} 0}^{1},\left\langle\Delta_{D}\left(\Gamma_{0}+\left(x^{1} d x^{2}-x^{2} d x^{1}\right) \otimes \frac{\partial}{\partial y^{1}}, \nabla^{0}\right)\left(j_{0}^{2} 0\right),\left.\frac{\partial}{\partial x^{1}}\right|_{0}\right\rangle\right\rangle .
$$

Because of the invariance of $\Delta_{D}$ with respect to exchanging $x^{1}$ and $x^{2}$ (i.e. with respect to the map $c: \mathbf{R}^{m, n} \rightarrow \mathbf{R}^{m, n}$ given by $c\left(x^{1}, x^{2}, \ldots, x_{m}, y\right)=$ $\left.\left(x^{2}, x^{1}, \ldots, x_{m}, y\right)\right)$, we get

$$
\begin{aligned}
& \left\langle Y_{1 \mid j_{0}^{2} 0}^{1},\left\langle\Delta_{D}\left(\Gamma_{0}+\left(x^{1} d x^{2}-x^{2} d x^{1}\right) \otimes \frac{\partial}{\partial y^{1}}, \nabla^{0}\right)\left(j_{0}^{2} 0\right),\left.\frac{\partial}{\partial x^{2}}\right|_{0}\right\rangle\right\rangle \\
& \quad=-\left\langle Y_{2 \mid j_{0}^{2} 0}^{1},\left\langle\Delta_{D}\left(\Gamma_{0}+\left(x^{1} d x^{2}-x^{2} d x^{1}\right) \otimes \frac{\partial}{\partial y^{1}}, \nabla^{0}\right)\left(j_{0}^{2} 0\right),\left.\frac{\partial}{\partial x^{1}}\right|_{0}\right\rangle\right\rangle .
\end{aligned}
$$

Consequently, the vector space of all possible values (27) is of dimension $\leq 1$. So, the vector space of all possible $\Delta_{D}$ is of dimension $\leq 2+K$, where $K$ is the dimension of the vector space of all possible $g$.

If $D=\mathcal{J}_{[i]}^{2}$ for $i=1,2$ is as in Example 3, then we have

$$
\begin{aligned}
& \left\langle\Delta_{\mathcal{J}_{[1]}^{2}}\left(\Gamma_{0}+\left(x^{1} x^{2} d x^{2}-\left(x^{2}\right)^{2} d x^{1}\right) \otimes \frac{\partial}{\partial y^{1}}, \nabla^{0}\right)\left(j_{0}^{2} 0\right),\left.\frac{\partial}{\partial x^{1}}\right|_{0}\right\rangle=0, \\
& \left\langle\Delta_{\mathcal{J}_{[1]}^{2}}\left(\Gamma_{0}+\left(x^{1} d x^{2}-x^{2} d x^{1}\right) \otimes \frac{\partial}{\partial y^{1}}, \nabla^{0}\right)\left(j_{0}^{2} 0\right),\left.\frac{\partial}{\partial x^{1}}\right|_{0}\right\rangle \\
& \quad=\mathcal{J}^{2}\left(x^{2} \frac{\partial}{\partial y^{1}}\right)\left(j_{0}^{2} 0\right), \\
& \left\langle\Delta_{\mathcal{J}_{[2]}^{2}}\left(\Gamma_{0}+\left(x^{1} x^{2} d x^{2}-\left(x^{2}\right)^{2} d x^{1}\right) \otimes \frac{\partial}{\partial y^{1}}, \nabla^{0}\right)\left(j_{0}^{2} 0\right),\left.\frac{\partial}{\partial x^{1}}\right|_{0}\right\rangle \\
& \quad=\mathcal{J}^{2}\left(\left(x^{2}\right)^{2} \frac{\partial}{\partial y^{1}}\right)\left(j_{0}^{2} 0\right), \\
& \left\langle\Delta_{\mathcal{J}_{[2]}^{2}}\left(\Gamma_{0}+\left(x^{1} d x^{2}-x^{2} d x^{1}\right) \otimes \frac{\partial}{\partial y^{1}}, \nabla^{0}\right)\left(j_{0}^{2} 0\right),\left.\frac{\partial}{\partial x^{1}}\right|_{0}\right\rangle=0, \\
& \Delta_{\mathcal{J}_{[i]}^{2}}\left(\Gamma_{0}, \nabla\right)(\rho)=0 \quad \text { for } \quad i=1,2
\end{aligned}
$$

for any $\rho \in\left(J^{2} \mathbf{R}^{m, n}\right)_{(0,0)}$ and any torsion free classical linear connection $\nabla \in Q_{\tau}\left(\mathbf{R}^{m}\right)$ such that $i d_{\mathbf{R}^{m}}$ is a $\nabla$-normal coordinate system with center 0 . By the flow argument we see that

$$
\begin{gathered}
\mathcal{J}^{2}\left(\left(x^{2}\right)^{2} \frac{\partial}{\partial y^{1}}\right)\left(j_{0}^{2} 0\right) \cong j_{0}^{2}\left(\left(x^{2}\right)^{2}\right) \mathcal{J}^{2}\left(\frac{\partial}{\partial y^{1}}\right)\left(j_{0}^{2} 0\right), \\
\mathcal{J}^{2}\left(x^{2} \frac{\partial}{\partial y^{1}}\right)\left(j_{0}^{2} 0\right) \cong j_{0}^{2}\left(x^{2}\right) \mathcal{J}^{2}\left(\frac{\partial}{\partial y^{1}}\right)\left(j_{0}^{2} 0\right),
\end{gathered}
$$

and then they are linearly independent.

Using the dimension argument and the formula (23), we deduce that there exist unique real numbers $t_{1}$ and $t_{2}$ and an $\mathcal{F} \mathcal{M}_{m, n}$-natural operator 
$D_{1}$ such that

$$
D=\left(1-t_{1}-t_{2}\right) D_{1}+t_{1} \mathcal{J}_{[1]}^{2}+t_{2} \mathcal{J}_{[2]}^{2}
$$

(the affine combination) and

$$
\Delta_{D_{1}}\left(\Gamma, \nabla^{0}\right)(\rho)=0
$$

for all $\rho \in\left(J^{2} \mathbf{R}^{m, n}\right)_{(0,0)}$ and all general connections $\Gamma$ on $\mathbf{R}^{m, n}$ such that the identity map $\psi=i d_{\mathbf{R}^{m, n}}$ is a $\left(\Gamma, \nabla^{0},(0,0), 3\right)$-quasi-normal fibred coordinate system on $\mathbf{R}^{m, n}$. The operator $D_{1}$ is uniquely determined if $t_{1}+t_{2} \neq 1$.

It remains to show that $D_{1}$ is of the form

$$
D_{1}=\mathcal{J}_{(A)}^{2}
$$

for a uniquely determined $\mathcal{M} f_{m}$-natural operator $A$ transforming torsion free classical linear connections $\nabla$ on $m$-manifolds $M$ into second order linear connections $A(\nabla): T M \rightarrow J^{2} T M$ on $M$, where $\mathcal{J}_{(A)}^{2}$ is as in Example 1.

We construct $A$ in the following way. Given a torsion free classical linear connection $\nabla$ on a $m$-manifold $M$ we define a tensor field $\tilde{A}(\nabla): M \rightarrow$ $T^{*} M \otimes S^{2} T^{*} M \otimes T M$ on $\mathrm{M}$ by

$$
\left\langle\tilde{A}(\nabla)_{\mid x}, \omega\right\rangle=p r_{1} \circ \Delta_{D_{1}}\left(\Gamma_{M}, \nabla\right)\left(j_{x}^{2}(f, 0, \ldots, 0)\right) \in T_{x}^{*} M \otimes S^{2} T_{x}^{*} M,
$$

where $\omega=d_{x} f \in T_{x}^{*} M, f: M \rightarrow \mathbf{R}, f(x)=0, \Gamma_{M}$ is the trivial general connection on the trivial bundle $M \times \mathbf{R}^{n} \rightarrow M$ and

$p r_{1}: T^{*} M \otimes S^{2} T^{*} M \otimes V\left(M \times \mathbf{R}^{n}\right)=T^{*} M \otimes S^{2} T^{*} M \otimes \mathbf{R}^{n} \rightarrow T^{*} M \otimes S^{2} T^{*} M$ is the projection onto the first factor.

The definition (36) is correct because

$$
\begin{aligned}
\Delta_{D_{1}}\left(\Gamma_{M}, \nabla\right)\left(j_{x}^{2}(f, 0, \ldots, 0)\right) & \in T^{*} M \otimes S^{2} T^{*} M \otimes V\left(M \times \mathbf{R}^{n}\right) \\
& \subset T^{*} M \otimes V J^{2}\left(M \times \mathbf{R}^{n}\right)
\end{aligned}
$$

as $\Delta_{D_{1}}\left(\Gamma_{M}, \nabla\right)\left(j_{x}^{2}(f, 0, \ldots, 0)\right)$ projects onto zero by

$$
i d_{T^{*} M} \otimes V \pi_{1}^{2}: T^{*} M \otimes V J^{2}\left(M \times \mathbf{R}^{n}\right) \rightarrow T^{*} M \otimes V J^{1}\left(M \times \mathbf{R}^{n}\right),
$$

where $\pi_{1}^{2}: J^{2} Y \rightarrow J^{1} Y$ is the jet projection. Indeed, in order to observe that $\Delta_{D_{1}}\left(\Gamma_{M}, \nabla\right)\left(j_{x}^{2}(f, 0, \ldots, 0)\right)$ projects onto zero, we can assume that $M=\mathbf{R}^{m}, x=0$ and $\psi=i d_{\mathbf{R}^{m, n}}$ is a $\left(\Gamma_{0}, \nabla,(0,0), 3\right)$-quasi-normal fibred coordinate system on $\mathbf{R}^{m, n}$ because of the $\mathcal{F} \mathcal{M}_{m, n}$-invariance of $\Delta_{D_{1}}$. From (26) for $\Delta_{D_{1}}$ instead of $\Delta_{D}$ we have $\Delta_{D_{1}}\left(\Gamma_{0}, \nabla^{0}\right)\left(j_{0}^{2}(f, 0, \ldots, 0)\right)=0$. Then using the invariance of $\Delta_{D_{1}}$ with respect to the homotheties and applying the homogeneous function theorem, we complete the observation.

Using the invariance of $\Delta_{D_{1}}$ with respect to the fiber homotheties $i d_{M} \times$ $t i d_{\mathbf{R}^{n}}$ and applying the homogeneous function theorem, we see that the value (36) depends linearly on $\omega$. Hence $\tilde{A}$ is really a tensor field.

Let

$$
A(\nabla):=A_{2}^{e x p}(\nabla)+\tilde{A}(\nabla): T M \rightarrow J^{2} T M
$$


be the second order connection corresponding to $\tilde{A}$. So, we have constructed an $\mathcal{M} f_{m}$-natural operator $A$ transforming torsion free classical linear connections $\nabla$ on $m$-manifolds $M$ into second order linear connections $A(\nabla): T M \rightarrow J^{2} T M$ on $M$.

We prove (35) as follows. Using the invariance of $A-A_{2}^{e x p}$ with respect to the homotheties and applying the homogeneous function theorem, we see that $A\left(\nabla^{0}\right)-A_{2}^{e x p}\left(\nabla^{0}\right)$ is the zero tensor field of type $T^{*} \otimes S^{2} T^{*} \otimes$ $T$. Therefore, we obtain (34) for $\Delta_{\mathcal{J}_{(A)}}$ instead of $\Delta_{D_{1}}$. Then using the condition (34), we get

$$
\begin{aligned}
& \left\langle Y_{22 \mid \rho}^{1},\left\langle\Delta_{D_{1}}(\Gamma, \nabla)(\rho),\left.\frac{\partial}{\partial x^{1}}\right|_{0}\right\rangle\right\rangle \\
& =\left\langle Y_{22 \mid \rho}^{1},\left\langle\Delta_{D_{\mathcal{J}_{(A)}}}(\Gamma, \nabla)(\rho),\left.\frac{\partial}{\partial x^{1}}\right|_{0}\right\rangle\right\rangle=g\left(\left(g_{l}^{1}\right),\left(\nabla_{i j ; k}^{l}\right)\right)
\end{aligned}
$$

for any $\rho \in\left(J^{2} \mathbf{R}^{m, n}\right)_{(0,0)}$, any general connection $\Gamma$ on $\mathbf{R}^{m, n}$ and any torsion free classical linear connection $\nabla$ on $\mathbf{R}^{m}$ such that the identity map $\psi=$ $i d_{\mathbf{R}^{m, n}}$ is a $(\Gamma, \nabla,(0,0), 3)$-quasi-normal coordinate system on $\mathbf{R}^{m, n}$, where

$$
\begin{aligned}
j_{(0,0)}^{2} \Gamma= & j_{(0,0)}^{2}\left(\Gamma_{0}+\sum_{i, j, k=1}^{m} \sum_{p=1}^{n} a_{k i j}^{p} x^{k} x^{i} d x^{j} \otimes \frac{\partial}{\partial y^{p}}\right. \\
& \left.+\sum_{i, j=1}^{m} \sum_{p, q=1}^{n} b_{q i j}^{p} y^{q} x^{i} d x^{j} \otimes \frac{\partial}{\partial y^{p}}+\sum_{i, j=1}^{m} \sum_{p=1}^{n} c_{i j}^{p} x^{i} d x^{j} \otimes \frac{\partial}{\partial y^{p}}\right)
\end{aligned}
$$

with coefficients $a_{k i j}^{p}, b_{q i j}^{p}$ and $c_{i j}^{p}$ satisfying (2),

$$
j_{0}^{1}(\nabla)=j_{0}^{1}\left(\left(\sum_{k=1}^{m} \nabla_{i j ; k}^{l} x^{k}\right)_{i, j, l=1}^{m}\right)
$$

for $\nabla_{i j ; k}^{l}=\nabla_{j i ; k}^{l} \in \mathbf{R}$ satisfying some "classical" conditions, $\rho$ is of the form

$$
\rho=j_{0}^{2}\left(\left(\sum_{i=1}^{m} g_{i}^{p} x^{i}+\sum_{i, j=1}^{m} h_{i j}^{p} x^{i} x^{j}\right)_{p=1}^{n}\right)
$$

for real numbers $g_{i}^{p}, h_{i j}^{p}=h_{j i}^{p}$ and $g$ is the bilinear map as in (23). Then we have (35) because any $\Delta_{D}$ (and then any $D$ ) is determined by the values (18).

If $D_{1}=\mathcal{J}_{\left(A_{1}\right)}^{2}$ for another $\mathcal{M} f_{m}$-natural operator $A_{1}$ (of the type as the one of $A$ ), then

$$
\left\langle\tilde{A}(\nabla)_{\mid x}, \omega\right\rangle=\left\langle\tilde{A}_{1}(\nabla)_{\mid x}, \omega\right\rangle
$$

for any torsion free classical linear connection $\nabla$ on $M$ and any $\omega \in T_{x}^{*} M, x \in$ $M$, where $\tilde{A}_{1}(\nabla)=A_{1}(\nabla)-A_{2}^{e x p}(\nabla): M \rightarrow T^{*} M \otimes S^{2} T^{*} M \otimes T M$ is the tensor field corresponding to $A_{1}(\nabla): T M \rightarrow J^{2} T M$. 
Because of $\mathcal{M} f_{m}$-invariance it is sufficient to show (39) in the case $M=$ $\mathbf{R}^{m}, x=0$ and the identity map $\psi=i d_{\mathbf{R}_{m, n}}$ is a $(\Gamma, \nabla,(0,0), 3)$-quasinormal fibred coordinate system on $\mathbf{R}_{m, n}$. It is not difficult. So, $A_{1}=A$, i.e. $A$ satisfying (35) is uniquely determined. The proof of Theorem 1 for $m \geq 2$ is complete.

If $m=1$, we proceed similarly as in the case $m \geq 2$. Therefore, $\Delta_{D}$ is uniquely determined by the values

$$
\begin{aligned}
& \left\langle Y_{\mid \rho}^{1},\left\langle\Delta_{D}(\Gamma, \nabla)(\rho),\left.\frac{\partial}{\partial x^{1}}\right|_{0}\right\rangle\right\rangle \in \mathbf{R} \\
& \left\langle Y_{1 \mid \rho}^{1},\left\langle\Delta_{D}(\Gamma, \nabla)(\rho),\left.\frac{\partial}{\partial x^{1}}\right|_{0}\right\rangle\right\rangle \in \mathbf{R} \\
& \left\langle Y_{11 \mid \rho}^{1},\left\langle\Delta_{D}(\Gamma, \nabla)(\rho),\left.\frac{\partial}{\partial x^{1}}\right|_{0}\right\rangle\right\rangle \in \mathbf{R}
\end{aligned}
$$

for all $\rho \in\left(J^{2} \mathbf{R}^{1, n}\right)_{(0,0)}$, all general connections $\Gamma$ on $\mathbf{R}^{1, n}$ and all torsion free classical linear connections $\nabla$ on $\mathbf{R}$ such that $\psi=i d_{\mathbf{R}^{1, n}}$ is a $(\Gamma, \nabla,(0,0), 3)$ quasi-normal fibred coordinate system on $\mathbf{R}^{1, n}$ over $\psi=i d_{\mathbf{R}}$. Then the operator $\Delta_{D}$ is uniquely determined by the values

$$
\left\langle Y_{11 \mid \rho}^{1},\left\langle\Delta_{D}(\Gamma, \nabla)(\rho),\left.\frac{\partial}{\partial x^{1}}\right|_{0}\right\rangle\right\rangle \in \mathbf{R} .
$$

If the identity map $\psi=i d_{\mathbf{R}^{1, n}}$ is a $(\Gamma, \nabla,(0,0), 3)$-quasi-normal fibred coordinate system, then $j_{(0,0)}^{2} \Gamma=j_{(0,0)}^{2}\left(\Gamma_{0}\right)$ and $j_{0}^{1}(\nabla)=j_{0}^{1}\left(\nabla^{0}\right)$ (as the curvature of $\nabla$ is zero). Consequently, $\Delta_{D}$ is determined by the values

$$
\left\langle Y_{11}^{1},\left\langle\Delta_{D}\left(\Gamma_{0}, \nabla^{0}\right)(\rho),\left.\frac{\partial}{\partial x^{1}}\right|_{0}\right\rangle\right\rangle \in \mathbf{R}
$$

for all $\rho \in J_{0}^{2}\left(\mathbf{R}, \mathbf{R}^{n}\right)_{0}$. But the values (40) are zero because of the similar arguments as in the proof of formula (23).

The proof of Theorem 1 is complete.

\section{REFERENCES}

[1] Doupovec, M., Mikulski, W., Holonomic extension of connections and symmetrization of jets, Rep. Math. Phys. 60 (2007), 299-316.

[2] Ehresmann, C., Sur les connexions d'ordre supérieur, Atti del V. Cong. del'Unione Mat. Italiana, 1955, Cremonese, Roma, 1956, 344-346.

[3] Kolář, I., Higher order absolute differentiation with respect to generalized connections, Differential Geometry, Banach Center Publ. 12, PWN-Polish Sci. Publ., Warszawa, 1984, 153-162.

[4] Kolář, I., Michor, P. W., Slovák, J., Natural Operations in Differential Geometry, Springer-Verlag, Berlin, 1993.

[5] Kolár, I., Prolongations of generalized connections, Differential Geometry (Budapest, 1979), Colloq. Math. Soc. János Bolyai, 31, North-Holland, Amsterdam, 1982, $317-$ 325. 
[6] Kolár, I., On the torsion free connections on higher order frame bundles, New Developments in Differential Geometry (Debrecen, 1994), Proceedings (Conference in Debrecen), Math. Appl., 350, Kluwer Acad. Publ., Dordrecht, 1996, 233-241.

[7] Kurek, J., Mikulski, W., On prolongations of projectable connections, Ann. Polon. Math. 101 (2011), no. 3, 237-250.

[8] Mikulski, W., On "special" fibred coordinates for general and classical connections, Ann. Polon. Math. 99 (2010), 99-105.

[9] Mikulski, W., Higher order linear connections from first order ones, Arch. Math. (Brno) 43 (2007), 285-288.

Mariusz Plaszczyk

Institute of Mathematics

Maria Curie-Skłodowska University

pl. M. Curie-Skłodowskiej 1

20-031 Lublin

Poland

e-mail: mariusz.piotr.plaszczyk@gmail.com

Received June 10, 2013 